\title{
Translocation of polyubiquitinated protein substrates by the hexameric Cdc48 ATPase
}

Zhejian Ji ${ }^{1 \$ *}$, Hao Li ${ }^{1 \$}$, Daniele Peterle ${ }^{2}$, Joao A. Paulo ${ }^{3}$, Scott B. Ficarro ${ }^{4,5}$, Thomas E. Wales ${ }^{2}$, Jarrod A. Marto ${ }^{4,5}$, Steven P. Gygi ${ }^{3}$, John R. Engen ${ }^{2}$, and Tom A. Rapoport ${ }^{1 *}$

${ }^{1}$ Howard Hughes Medical Institute and Department of Cell Biology, Harvard Medical School, 240 Longwood Avenue, Boston, Massachusetts 02115, USA.

2Department of Chemistry and Chemical Biology, Northeastern University, Boston, MA, USA.

${ }^{3}$ Department of Cell Biology, Harvard Medical School, 240 Longwood Avenue, Boston, Massachusetts 02115, USA.

${ }^{4}$ Department of Cancer Biology, Department of Oncologic Pathology, and Blais Proteomics Center, Dana-Farber Cancer Institute, Boston, MA 02115, USA;

${ }^{5}$ Department of Pathology, Brigham and Women's Hospital, Harvard Medical School, Boston, MA 02115, USA.

\$These authors contributed equally.

*Corresponding authors:

Zhejian Ji and Tom Rapoport, Howard Hughes Medical Institute and Department of Cell Biology, Harvard Medical School, 240 Longwood Avenue, Boston, Massachusetts 02115, USA.

Emails: Zhejian Ji@hms.harvard.edu and tom rapoport@hms.harvard.edu 


\section{SUMMARY}

3 The hexameric Cdc48 ATPase (p97 or VCP in mammals) cooperates with its cofactor Ufd1/Npl4

4 to extract polyubiquitinated proteins from membranes or macromolecular complexes for

5 degradation by the proteasome. Here, we clarify how the Cdc48 complex unfolds its substrates

6 and translocates polypeptides with branchpoints. The Cdc48 complex recognizes primarily

7 polyubiquitin chains, rather than the attached substrate. Cdc48 and Ufd1/Npl4 cooperatively

8 bind the polyubiquitin chain, resulting in the unfolding of one ubiquitin molecule (initiator).

9 Next, the ATPase pulls on the initiator ubiquitin and moves all ubiquitin molecules linked to its

10 C-terminus through the central pore of the hexameric double-ring, causing transient ubiquitin

11 unfolding. When the ATPase reaches the isopeptide bond of the substrate, it can translocate

12 and unfold both $\mathrm{N}$ - and $\mathrm{C}$-terminal segments. Ubiquitins linked to the branchpoint of the

13 initiator dissociate from Ufd1/Npl4 and move outside the central pore, resulting in the release

14 of unfolded, polyubiquitinated substrate from Cdc48.

15

16 Key words: AAA ATPase, translocation, ubiquitin, p97, VCP, Npl4, Ufd1, unfolding 
The Cdc48 ATPase in Saccharomyces cerevisiae and its p97 (VCP) orthologs in metazoans extract polyubiquitinated substrate polypeptides from membranes or macromolecule complexes and generally deliver them to the proteasome for degradation (Bodnar and

23 Rapoport, 2017a; van den Boom and Meyer, 2018). For example, in endoplasmic reticulum

24 (ER)-associated protein degradation (ERAD), Cdc48/p97 pulls misfolded, polyubiquitinated

25 proteins out of the ER membrane for their subsequent degradation (Wu and Rapoport,

26 2018). Cdc48/p97 consists of an $\mathrm{N}$-terminal $\mathrm{N}$ domain and two ATPase domains (D1 and D2)

27 (Bodnar and Rapoport, 2017a) (Figure S1A). Six molecules of the ATPase form a double-ring structure with a central pore. In ERAD and many other processes, Cdc48/p97 cooperates with the heterodimeric Ufd1/Npl4 (UN) cofactor. Like Cdc48/p97, UN is found in all eukaryotic cells and is essential for their viability. UN recruits substrates to the Cdc48/p97 ATPase by interacting with the attached K48-linked polyubiquitin chain. Subsequently, the ATPase uses ATP hydrolysis to translocate the polypeptide through the central pore, thereby causing its unfolding (Blythe et al., 2017; Bodnar and Rapoport, 2017b). The critical role of human p97 in protein quality control is highlighted by mutations that cause neurodegenerative proteopathies (Johnson et al., 2010; Kimonis et al., 2008; Watts et al., 2004). p97 is also an important cancer drug target, as inhibitors suppress the proliferation of multiple tumors (Anderson et al., 2015; Le Moigne et al., 2017). Despite its importance, the mechanism by which Cdc48/p97 processes its substrates remains poorly understood.

In previous work, we determined cryo-EM structures of Cdc48 in complex with the UN cofactor and a polyubiquitinated model substrate (Twomey et al., 2019) (Figure S1A). The structures represented an initiation state of substrate processing prior to ATP hydrolysis. The D1 and D2 domains formed stacked hexameric rings, while Npl4 formed a tower-like structure above the D1 ring (Figure S1A). Most of the Ufd1 molecule was invisible. Three consecutive ubiquitin molecules of the substrate-attached polyubiquitin chain were seen. Two folded ubiquitin molecules (Ub1 and Ub2) were bound to the top of the Npl4 tower, and one ubiquitin molecule

47 was unfolded (the "initiator ubiquitin"), with its N-terminal segment traversing the central 
pores of both ATPase rings and a subsequent segment bound to a groove in Npl4 (Figure S1A). We hypothesized that $\mathrm{Cdc} 48$ begins polypeptide translocation by pulling on the $\mathrm{N}$-terminal segment of the initiator ubiquitin, because this segment interacts with the D2 pore loops and the D2 ATPases are responsible for polypeptide movement (Bodnar and Rapoport, 2017b).

Substrate processing by the Cdc48 complex can be divided into three phases--substrate

54 recruitment, translocation, and release-- which are all poorly understood. Our previous in vitro experiments suggested that a polyubiquitin chain is sufficient to initiate translocation (Bodnar and Rapoport, 2017b), while the actual substrate to which the chain is attached plays no role. However, it remained unclear whether in vivo the ATPase complex indeed indiscriminately processes all polyubiquitinated proteins, particularly because this would raise the possibility that all such substrates are unfolded by the ATPase, and that Cdc48/p97 and the $26 \mathrm{~S}$ proteasome compete with one another for polyubiquitinated proteins. How the ubiquitin chain is recognized by the Cdc48 complex is also unclear. The most mysterious aspect concerns the unfolding of the initiator ubiquitin molecule (Twomey et al., 2019). Ubiquitin is extremely stable, and yet it can be unfolded by a simple binding reaction, without the need for ATP hydrolysis.

Many aspects of the translocation process also remain unclear. If $\mathrm{Cdc} 48$ begins translocation by pulling on the initiator ubiquitin, it would need to translocate all ubiquitin molecules positioned between the initiator and substrate (proximal ubiquitins) before it can unfold the substrate

(Figure S1A). This implies that the ATPase translocates branched polypeptide chains, as each ubiquitin molecule is linked by an isopeptide bond through its C-terminus to K48 of another ubiquitin molecule or to a Lys residue of the substrate. It is unknown how Cdc48/p97 deals with

72 such branchpoints, i.e. whether it translocates and unfolds both polypeptide branches or only

73 one of them. The ability to process branched polypeptides sets the Cdc48/p97 ATPase apart

74 from most other known ATPases, which translocate only linear polypeptide chains. For

75 example, the $26 \mathrm{~S}$ proteasome recognizes polyubiquitin chains similarly to the $\mathrm{Cdc} 48 / \mathrm{p} 97$

76 ATPase, but it cleaves them off before translocating and degrading an unbranched substrate 
77 polypeptide (Greene et al., 2020). Finally, it is unclear how substrate is released from the

$78 \mathrm{Cdc} 48 / \mathrm{p} 97$ complex after completion of translocation, so that it can be transferred to the

79 proteasome.

81 Here, we show that the Cdc48 ATPase complex indeed shows little substrate specificity,

82 processing the majority of polyubiquitinated substrates in a cell. We clarify the mechanisms of

83 all three phases of substrate processing by showing how the ATPase complex unfolds the

84 initiator ubiquitin, how it translocates polypeptides with branchpoints, and how it releases its

85 polyubiquitinated substrates. Our work provides a comprehensive model for protein unfolding

86 by the Cdc48 ATPase and suggests that the ATPase acts both upstream and downstream of the

87 proteasome.

\section{RESULTS}

The Cdc48 complex recognizes primarily the polyubiquitin chain

93 We first tested in S. cerevisiae cells whether the Cdc48/UN complex binds equally well to all

94 polyubiquitinated proteins or whether it processes only a subset of them. We used quantitative

95 MS to compare the proteome carrying K48-linked polyubiquitin chains with the proteome

96 associated with the Cdc48/UN complex. The experiments were performed in the absence or

97 presence of the proteasome inhibitor bortezomib in cells lacking the drug exporter Pdr5

98 (Fleming et al., 2002).

100 To enrich for proteins bound to the Cdc48/UN complex, we expressed FLAG-tagged Npl4 or 101 Ufd1 (Npl4-FLAG and Ufd1-FLAG, respectively) in yeast cells, and subjected cell lysates to 102 immunoprecipitation (IP) with FLAG antibodies bound to beads (Figure 1A). Pulling on UN, 103 rather than $\mathrm{Cdc48}$, avoids the co-purification of other cofactors and their associated substrates.

104 The beads were then treated with an excess of trypsin-resistant tandem ubiquitin-binding 105 entity (TR-TUBE) (Tsuchiya et al., 2017), resulting in the transfer of polyubiquitinated proteins 
106

107

108

109

110

111

112

113

114

from the Cdc48 complex to TUBE. The samples were subjected to trypsin digestion and the resulting peptides labeled with Tandem Mass Tags (TMT). The use of trypsin-resistant TUBE prevented the interference of abundant TUBE peptides in the subsequent MS analysis. Proteins carrying K48-linked polyubiquitin chains were enriched by incubating cell lysates with biotinylated TUBE-K48 (BioTUBE ${ }^{\mathrm{K} 48}$ ), a protein that recognizes specifically K48-linked polyubiquitin chains, followed by incubation with streptavidin beads (K48 IP) (Figure 1A). Bound polyubiquitinated proteins were again eluted with TR-TUBE, digested with trypsin, and subjected to TMT labeling. Labeled peptides from all samples were mixed and subjected to tandem MS. For each substrate protein detected, we determined its relative abundance in the FLAG IP versus K48 IP (Cdc48/K48 ratio). With either Npl4-FLAG or Ufd1-FLAG pull-downs, most identified substrate proteins have approximately the same Cdc48/K48 ratio (Figure 1B; 1C), consistent with $\mathrm{Npl} 4$ and $\mathrm{Ufd} 1$ being stoichiometric components of the Cdc48/UN complex. Treatment with bortezomib did not significantly affect the Cdc48/K48 ratios (Figures 1D; 1E), despite the large accumulation of polyubiquitinated proteins in bortezomib-treated cells (Figure S1B). Averaging Cdc48/K48 ratios for each protein shows that $91 \%$ of all identified proteins (795 out of 873 ) had ratios between 0.3 and 3, i.e, did not drastically differ in their enrichment by pulling on the Cdc48 complex or K48-linked ubiquitin chains (Figure 1F). Thus, the ATPase complex recognizes primarily the polyubiquitin chain and has little specificity for the attached substrate.

\section{Cdc48-facilitated ubiquitin unfolding}

We next used in vitro experiments to analyze the molecular mechanism of substrate processing by the Cdc48 ATPase complex, first focusing on the initiation stage, during which a ubiquitin molecule is unfolded without the need for ATP hydrolysis. To follow substrate and ubiquitin unfolding, we performed hydrogen/deuterium exchange (HDX) mass spectrometry (MS) with purified Cdc48 complex and a polyubiquitinated model substrate. The substrate contained a degron sequence derived from the $\mathrm{N}$-end rule pathway (Chau et al., 1989) fused to the fluorescent protein Dendra (Kaberniuk et al., 2017) (Figure 2A). The fusion protein was irradiated with UV light, which leads to photoconversion from green to red fluorescence and 
cleavage of the Dendra polypeptide into two fragments that remain non-covalently associated with one another. The irradiated fusion protein was then incubated with purified ubiquitination enzymes to attach a single chain of 10-25 K48-linked ubiquitin molecules to a Lys residue in the $\mathrm{N}$-terminal segment, resulting in Ub(n)-Dendra (Bodnar and Rapoport, 2017b) (Figure 2A).

We first performed HDX experiments after incubating photoconverted Ub(n)-Dendra with Cdc48 complex in the presence of ADP, i.e. conditions in which Dendra is not translocated by the ATPase and remains folded, while the initiator ubiquitin should be unfolded. Deuterium was then added and HDX was followed for different time periods (Figure 2B). The reaction was quenched by low $\mathrm{pH}$, and the proteins proteolytically cleaved. Peptides derived from ubiquitin and Dendra were analyzed by MS and the extent of deuterium labeling determined. Analysis of ubiquitin peptides showed very little Cdc48 complex-dependent labeling at early time points of HDX (Figure 2C). With time, however, significantly more HDX was observed in the presence of Cdc48 complex, consistent with the complex causing ubiquitin unfolding. Deuteration was particularly strong in an $\mathrm{N}$-terminal ubiquitin segment and a region in the middle of the sequence, which in the cryo-EM structure interact with Cdc48 and the Npl4 groove, respectively (Twomey et al., 2019). Ultimately, almost the entire ubiquitin population became labeled (Figure S2A), suggesting that most ubiquitin molecules in a polyubiquitin chain can serve as initiators and undergo Cdc48 complex-induced cycles of unfolding and refolding. Dendra peptides showed little labeling in the presence of ADP (Figure 2D). In contrast, when the preincubation was performed in the presence of ATP, so that substrate was unfolded before HDX (Figure 2B), two N-terminal $\beta$-strands of Dendra were maximally deuterated even at the shortest labeling time (Figure 2D). This is consistent with the expectation that, during the preincubation, the N-terminal fragment of Dendra (residues 1-192) is translocated through the central pore, while the C-terminal fragment (residues 193-355) stays behind, thus separating the two $\beta$-strands and allowing their deuteration. Surprisingly, the presence of ATP during the unfolding reaction had no effect on the subsequent deuteration of ubiquitin peptides (Figure 2C). As shown below, ubiquitin molecules are actually translocated through the central pore and transiently unfolded, but they refold and again serve as initiators (Figure 7A). 
To directly measure ubiquitin unfolding, we mutated lle3 of ubiquitin to Cys (Ub ${ }^{13 \mathrm{C}}$ ). According to the crystal structure of ubiquitin (PDB code $1 U B Q$ ), the side chain of this residue is buried in

167 the interior of the folded molecule and should only be exposed after unfolding. Unfolding was

168 examined in the presence of ADP by modification of $\mathrm{Ub}^{13 \mathrm{C}}$ with a maleimide-conjugated

169 fluorescent dye (Dy-maleimide), employing Ub(n)-Dendra substrate that lacks other cysteines

170 (Figure 2E). Some weak modification occurred even in the absence of Cdc48 or its cofactors

171 (Figure 2E; lane 1), suggesting occasional spontaneous unfolding of ubiquitin. Ufd1 or Npl4

172 alone had little effect (lanes 2 and 3), but together moderately stimulated modification (lane 5).

173 Modification was strongest when Cdc48 was also present (lane 8). Thus, all components of the

174 Cdc48 complex are required for efficient ubiquitin unfolding.

175

176 Cooperation of the Cdc48 components was also seen in binding experiments (Figure 2F). For

177 these experiments, we employed a fusion of the N-end-rule degron with super-folder GFP

178 (sfGFP) and attached a fluorescent dye to a Cys in the N-terminal segment for better detection

179 (Ub(n)-Dy-sfGFP) (Bodnar and Rapoport, 2017b). The Cdc48 complex was captured through

180 FLAG-tagged Npl4 and FLAG-antibody beads, and bound proteins analyzed by SDS-PAGE (Figure

181 2F). Binding of Ub(n)-Dy-sfGFP was strongest when all components were present (lane 12).

182 Much less binding was seen in the absence of Cdc48 or Ufd1 (lanes 10 and 11). Quantification

183 of the pull-down efficiencies indicated that about $50 \%$ of all fully assembled Cdc48 complexes

184 contain bound Ub(n)-Dy-sfGFP. A stimulatory effect of Cdc48 on substrate binding was also

185 seen when a streptavidin-binding peptide (SBP) tag was attached to Ufd1 and the pull-down

186 experiments were performed with streptavidin beads (Figure S2B). These results show that

$187 \mathrm{Npl} 4$ and Ufd1 are not sufficient for optimal binding of polyubiquitinated substrates. Previous

188 experiments suggested that they contain all known ubiquitin binding activity (Park et al.,

189 2005; Sato et al., 2019), but our experiments reveal that when the cofactor components are

190 present at equimolar concentrations, the Cdc48 ATPase greatly contributes to substrate

191 binding.

192 


\section{Insertion of the $\mathrm{N}$-terminal segment of the initiator ubiquitin into the $\mathrm{Cdc} 48$ ring}

The cryo-EM structure raised the possibility that Cdc48 stimulates substrate binding by

capturing the $\mathrm{N}$-terminal segment of the initiator ubiquitin in the central pore of the D1 ring.

Indeed, a truncated Cdc48 protein containing only the N and D1 domains (Cdc48 ${ }^{\mathrm{ND} 1}$ ) stimulated

ubiquitin unfolding in the $\mathrm{Ub}^{\mathrm{ISC}}$-labeling assay (Figure $\mathbf{2 E}$, lanes 11-20) and the binding of polyubiquitinated substrate to the ATPase complex (Figure S2C). Capture of the unfolded ubiquitin in the D1 pore is further supported by site-specific crosslinking experiments, in which the photoreactive probe $\mathrm{p}$-benzoyl-phenylalanine (Bpa) was incorporated by amber-codon suppression (Chin et al., 2002) into the D1 ring of either full-length Cdc48 or Cdc48 ${ }^{\mathrm{ND} 1}$; in both cases, crosslinks to polyubiquitinated substrate were observed in ADP or ATP (Figure S2D).

203 Taken together, these results show that the D2 ATPase ring is not required for ubiquitin 204 unfolding and binding.

Next, we tested whether the insertion of the initiator ubiquitin segment requires the pore loops of the D1 ring. Binding experiments with $\mathrm{Ub}(\mathrm{n})$-Dy-Dendra showed that a Cdc $48^{\mathrm{ND} 1}$ mutant lacking the pore loop residues ( $\triangle \mathrm{D} 1 \mathrm{Loops})$ had a reduced affinity compared to wild-type $\mathrm{Cdc48} 8^{\mathrm{ND} 1}$ or a mutant of $\mathrm{Cdc} 48^{\mathrm{ND} 1}$ that lacks ATPase activity (E315A) (Figure 3A; lane 5 versus lanes 4 and 6). The importance of the D1 pore loops is further highlighted by substrate

211 unfolding experiments that utilized photoconverted $\mathrm{Ub}(\mathrm{n})$-Dy-Dendra; in this assay, the two

212 fragments of photoconverted Dendra are separated during the unfolding reaction, which

213 results in a loss of fluorescence. Almost complete substrate unfolding was observed with wild-

214 type Cdc48 complex or the E315A mutant (Figure 3B), consistent with D1 ATPase activity not

215 being required (Blythe et al., 2017; Bodnar and Rapoport, 2017b). However, mutant Cdc48

216 lacking the D1 pore loops was completely inactive (Figure 3B), highlighting the importance of an

217 interaction between the D1 pore loops and the N-terminal segment of the initiator ubiquitin

218 molecule.

220 Given the substantial length of the N-terminal segment of the initiator ubiquitin and its 221 extended conformation in the cryo-EM structure, it seemed possible that it would enter the 
222 central pore of the D1 ring from the side. To test this possibility, we generated a Cdc48

223 hexamer in which all subunits are disulfide-crosslinked to their neighbors so that lateral entry

224 would be prevented (Figure 3C). This was achieved by introducing cysteines at S286 and R333,

225 which are located at the interface of neighboring protomers close to the D1 pore entrance

226 (Figure S2E). Treatment of this mutant ( $\left.\mathrm{Cdc} 48^{2 \mathrm{Cys}}\right)$ with an oxidant resulted in efficient

227 crosslinking of all six protomers of the Cdc48 hexamer (Figure 3D; bottom panel). The

228 crosslinked $\mathrm{Cdc} 48^{2 \mathrm{Cys}}$ complex stimulated binding of polyubiquitinated substrate to the UN

229 cofactor to a similar extent as the non-crosslinked complex (Figure 3D; upper most panel; lane

2304 versus lanes 3 and 5). Thus, the $\mathrm{N}$-terminal segment of the initiator ubiquitin likely enters the

231 pore from the top of a closed D1 ring, rather than from the side. Interestingly, the crosslinked

$232 \mathrm{Cdc}^{2 \mathrm{2Cys}}$ hexamer was inactive in substrate unfolding, but was re-activated upon reduction of

233 the disulfides (Figure 3E). Given that ATP hydrolysis in the D1 protomers is not required for

234 substrate unfolding (Figure 3B; E315A mutant), we consider it unlikely that crosslinking simply

235 prevented movements of the D1 protomers; rather, a closed hexameric D1 ring may prevent

236 the K48-branchpoint of the initiator ubiquitin from moving through the central pore (see

237 below).

To further test whether the $\mathrm{N}$-terminus of the initiator ubiquitin enters a closed D1 ring, we generated a substrate in which the ubiquitin chain in photoconverted $\mathrm{Ub}(\mathrm{n})$-Dendra was replaced with a chain of Dendra-ubiquitin fusions (Figure 3F); the bulky Dendra domains should prevent the $\mathrm{N}$-termini of all ubiquitin molecules from entering the ATPase ring. Indeed, no unfolding was observed with this substrate (Figure 3F). However, when Dendra was removed by taking advantage of a TEV protease cleavage site between the fusion partners, unfolding was restored (Figure 3F). These experiments show that a free $\mathrm{N}$-terminus is required for ubiquitin to serve as initiator. Interestingly, when the small ubiquitin-like modifier (SUMO) protein was used as $\mathrm{N}$-terminal fusion partner of ubiquitin, slow unfolding was observed (which was accelerated after cleavage of SUMO by the Ulp1 protease) (Figure S2F). A likely explanation is that a SUMO molecule can serve as initiator, albeit not as efficiently as ubiquitin. 
251 Taken together, our results show that the Cdc48 ATPase has a hitherto unappreciated role in

252 substrate recruitment: it uses the pore loops of the D1 ring to capture the N-terminal segment

253 of the unfolded initiator ubiquitin inside the central pore, thereby augmenting the binding of

254 ubiquitin molecules to the Cdc48/UN complex.

Ubiquitin unfolding requires the cofactors Npl4 and Ufd1

257 Next, we tested the role of the cofactors in ubiquitin unfolding. We first made several

258 mutations in the Npl4 groove (Figure S3A), which according to the cryo-EM structure (Twomey

259 et al., 2019), accommodates residues 23 to 48 of the initiator ubiquitin. Mutation of amino

260 acids at the bottom or top of Npl4's groove (Figure S3A; inset i) reduced the binding of Ub(n)-

261 Dy-Dendra to the Cdc48 complex, whereas mutation of residues contacting the kink of the

262 initiator ubiquitin (Figure S3A) had little effect (Figure S3B). Binding of Ub(n)-Dy-Dendra to the

263 mutants showed a correlation with the initial rate of Dendra unfolding (Figure S3C). Ub(n)-Dy-

264 Dendra binding also correlated with the efficiency of polypeptide insertion into the central pore

265 of Cdc48, as demonstrated by site-specific photo-crosslinking experiments with Bpa probes in

266 the D2 ring of Cdc48 (Figure S3D). These results indicate that the Npl4 groove plays an

267 important role in the binding of the initiator ubiquitin and subsequent substrate processing.

269 We next probed the interaction of Npl4 with the two folded ubiquitin molecules Ub1 and Ub2

270 visible in the cryo-EM structure (Figure S3A; insets ii and iii). Mutations designed to disrupt the

271 interaction with Ub2 (Sato et al., 2019) only moderately reduced Ub(n)-Dy-sfGFP binding

272 (Figure S3E; lanes 5-7 versus 4). Two of the four mutations introduced at the interface to Ub1

273 also attenuated binding (lanes 8-11). However, surprisingly, all mutations only slightly reduced

274 substrate unfolding (Figure S3F). Thus, the interaction of Npl4 with the folded ubiquitin

275 molecules is not crucial, consistent with the low sequence conservation of Npl4 in the

276 interacting regions (Twomey et al., 2019). These results suggest that Ufd1 may be more

277 important for the interaction with folded ubiquitin molecules. 
279 To test the role of Ufd1, we deleted the N-terminal, ubiquitin-binding UT3 domain (Park et al.,

280 2005) (Figure S3G). Indeed, substrate recruitment to the Cdc48 complex was completely

281 abolished (Figure S3H; lane 4 versus 5). The same result was obtained when photocrosslinking

282 of polyubiquitinated substrate to Cdc48's D2 ring was tested (Figure S3I; lane 4 versus 3), or

283 when the unfolding of photoconverted Dendra was measured (Figure S3J). Thus, the ubiquitin

284 binding activity of the UT3 domain of Ufd1 plays an essential role in substrate recruitment.

285 Taken together, these results show that the ubiquitin binding activities of the UN cofactor

286 promote the unfolding of the initiator ubiquitin and substrate recruitment to the Cdc48 ATPase.

Translocation of the initiator ubiquitin

289 Next, we investigated the translocation phase. We first tested whether translocation begins

290 with the Cdc48 ATPase pulling on the initiator ubiquitin. In this case, the initiator segment

291 originally bound to the Npl4 groove should be dislodged, which in turn should lead to the

292 displacement of Ub1 and Ub2 from the top of the Npl4 tower; if these ubiquitin molecules

293 cannot be released from their binding sites, translocation and substrate unfolding should be

294 prevented (Figure S4A). To test this prediction, we incorporated photoreactive Bpa probes into

295 the Npl4 groove and the top of the Npl4 tower by amber-codon suppression (Figure 4A). The

296 Bpa-containing Npl4 mutants were mixed with photoconverted Ub(n)-Dendra, Ufd1, and Cdc48,

297 and the mixtures were irradiated with UV light in the absence of nucleotides; ATP was then

298 added and the unfolding of Dendra monitored. All tested positions could crosslink to

299 polyubiquitinated substrate (Figure S4B), and all crosslinked complexes showed a reduced

300 ability to unfold substrate (Figure 4B). In contrast, irradiated mixtures containing wild-type Npl4

301 and all non-irradiated samples showed unperturbed unfolding kinetics (Figure 4B). In general,

302 there was a good correlation between the crosslinking yield and inhibition of the initial

303 unfolding rate (Figure 4C), indicating that the crosslinked complexes were entirely inactive.

304 These results show that translocation is prevented when the ubiquitin molecules cannot be

305 released from $\mathrm{Npl4}$, consistent with translocation beginning with the $\mathrm{N}$-terminal segment of the

306 initiator ubiquitin and causing the initiator ubiquitin, Ub1, and Ub2 to be dislodged from their

307 original binding sites on Npl4. 
Translocation of polypeptide branchpoints by the Cdc48 ATPase

310 The next important event happens when the K48 branchpoint of the initiator ubiquitin (called 311 type I) enters the ATPase rings (Figure 5A), as the ATPase could in principle translocate either 312 polypeptide branch through its central pore. Our unfolding experiments with Ub(n)-Dendra 313 (e.g. Figure 3B) indicate that Cdc48 can move towards the C-terminus of the initiator ubiquitin, 314 as this is the direction in which it can reach the substrate that is either directly attached to the 315 C-terminus or separated from the initiator by proximal ubiquitin molecules (Figure 5A).

317 To test whether Cdc48 can also translocate in the other direction when it encounters the K48 318 branchpoint of the initiator and therefore unfold distal ubiquitins, we generated a K48-linked 319 polyubiquitin chain with photoconverted Dendra attached to the most distal ubiquitin molecule 320 (Figure 5B; Dendra-Dist). This was achieved by performing a ubiquitination reaction with a 321 mixture of wild-type ubiquitin ( $\mathrm{Ub}^{\mathrm{WT}}$ ) and a fusion of Dendra to the K48R mutant of ubiquitin 322 (Dendra-U $\mathrm{b}^{\mathrm{K} 48 \mathrm{R}}$ ), using a purified fusion enzyme consisting of the RING finger domain of the 323 ubiquitin ligase gp78 and the conjugating enzyme Ube2G2 (Blythe et al., 2017);

324 incorporation of $\mathrm{Ub}^{\mathrm{K} 48 \mathrm{R}}$ terminates chain elongation, resulting in Dendra-Ub ${ }^{\mathrm{K} 48 \mathrm{R}}$ capping the 325 ubiquitin chains. Dendra-Dist was able to bind to the Cdc48 complex (Figure S4C), but addition of ATP did not cause any reduction of Dendra fluorescence (Figure $5 \mathrm{C}$ ), indicating that Cdc48

327 does not unfold distal ubiquitin molecules. As a control, we generated a similar substrate, but 328 with photoconverted Dendra at the proximal side of the initiator ubiquitin (Figure 5B; Dendra$329 \operatorname{Prox}(\mathrm{C}))$. Like Ub(n)-Dendra (Figure 3B), Dendra-Prox(C) substrate was efficiently unfolded 330 (Figure 5 C), again showing that proximal ubiquitins are translocated through the central pore of 331 Cdc48.

333 The next crucial event happens when the ATPase reaches the C-terminus of the initiator, as it 334 encounters another branchpoint (called type II), either K48 of another ubiquitin molecule or a 335 Lys residue of the substrate. This branchpoint is fundamentally different from the K48 336 branchpoint of the initiator (type I), as Cdc48 is now pulling on the isopeptide bond of a folded 
protein, rather than on the peptide bond of an unfolded ubiquitin segment protein (Figure 5A). Again, we asked whether Cdc48 translocates one or both branches through its central pore. Because photoconverted Dendra was attached to the C-terminus of the most proximal ubiquitin molecule in Ub(n)-Dendra, its unfolding indicates that the ATPase can translocate from the type II branchpoint towards the C-terminus of the substrate. To test whether Cdc48 can also translocate in the other direction, we fused photoconverted Dendra to the $\mathrm{N}$-terminus of the ubiquitin mutant $\mathrm{Ub}^{\mathrm{G} 76 \mathrm{~V}}$ and attached a K48-linked ubiquitin chain to Ub ${ }^{\mathrm{G} 76 \mathrm{~V}}$ (Figure 5B; Dendra$\operatorname{Prox}(\mathrm{N}))$; the G76V mutation ensures that the fusion protein is at the proximal end of the ubiquitin chain and that the $\mathrm{K} 48$ of $\mathrm{Ub}^{\mathrm{G} 76 \mathrm{~V}}$ is a type II branchpoint. Dendra was efficiently unfolded by Cdc48 (Figure $5 \mathrm{C}$ ), demonstrating that, in contrast to the type I branchpoint, type II

347 allows bidirectional translocation towards either the $\mathrm{N}$ - or C-terminus. One possible explanation for the selectivity at type I branchpoints is that Cdc48 cannot unfold ubiquitin when it pulls on its C-terminus. To test this possibility, we generated a substrate that contained Dendra fused to $\mathrm{Ub}^{\mathrm{K} 48 \mathrm{R}}$ at the $\mathrm{N}$-terminus of a polyubiquitin chain (Figure 5B; Dendra-Ub $\mathrm{B}^{\mathrm{K} 48 \mathrm{R}}$-Prox $(\mathrm{N})$ ). In this case, translocation can only be initiated within the ubiquitin chain, and when Cdc48 reaches $\mathrm{Ub}^{\mathrm{K} 48 \mathrm{R}}$ (blue), it has to pull on the C-terminus of this ubiquitin molecule before it can unfold

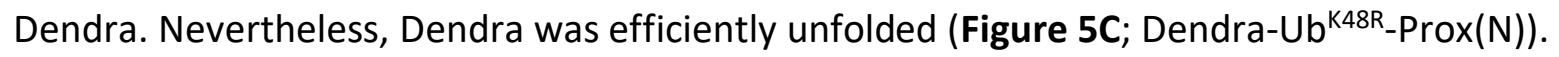
Thus, Cdc48 can unfold ubiquitin when it pulls on its C- terminus, but not at type I branchpoints.

To further test whether Cdc48 can translocate in either direction when it encounters a type II branchpoint, we generated fusion proteins in which ubiquitin is flanked by sfGFP and photoconverted Dendra (Dendra-Ub(n)-sfGFP and sfGFP-Ub(n)-Dendra) (Figures 5D; 5E). Both substrates were unfolded by the $\mathrm{Cdc} 48$ complex. Translocation of these substrates cannot be initiated by the ubiquitin molecule in the fusion protein because its $\mathrm{N}$-terminus is blocked by a

361 bulky protein (see Figure 3F). Rather, one of the ubiquitin molecules in the attached chain

362 (shown in purple) must have served as initiator, and translocation must have proceeded 363 through the ubiquitin in the fusion protein to Dendra. These results confirm that Cdc48 can 364 translocate towards either the $\mathrm{N}$ - or C-terminus when it encounters the isopeptide bond of the 365 substrate-attached ubiquitin molecule. Taken together, our results indicate that at the type I 
branchpoint of the initiator ubiquitin, Cdc48 selectively translocates the C-terminal segment through the central pore, but at subsequent type II branchpoints, it can move either polypeptide branch through the pore.

\section{Release of polyubiquitinated substrate from $\mathrm{Cdc} 48$}

371 Finally, we investigated whether the polyubiquitinated substrate is released from the Cdc48

372 complex after its translocation through the central pore. Ub(n)-Dendra was associated with the

373 Cdc48 complex, regardless of whether the incubation was performed in ADP or ATP (Figure 6A,

374 lane 5 versus 6), i.e. whether or not Dendra was folded or unfolded (Figure S5A). Thus, fully

375 translocated substrate was either not released from the Cdc48 complex or re-associated with it

376 (Figure 6B; scenarios a and b, respectively). Substrate release would be prevented if the distal

377 ubiquitins stayed on the cis side of the ATPase ring, such that proximal and distal ubiquitins

378 would end up on opposite sides of the ATPase rings, with a connecting polypeptide segment

379 spanning the pore (Figure 6B; scenario a). On the other hand, if the distal ubiquitins moved

380 outside the pore, while the K48 branchpoint of the initiator itself moved through the pore, at

381 the end of translocation all ubiquitin molecules and substrate would be released from the

382 Cdc48 complex and could re-associate to re-generate an initiation state, but this time with

383 unfolded substrate (Figure 6B; scenario b).

To test whether the final state resembles an initiation state, we took advantage of the fact that polyubiquitinated substrate cannot bind to the Cdc48 complex without the UT3 domain of Ufd1

(Figure S3H). We generated a Cdc48 complex, in which Ufd1 contains a TEV cleavage site

388 following its UT3 domain (Ufd1 ${ }^{\mathrm{TEV}}$ ), a complex that was active in Dendra unfolding (Figure S5B).

$389 \mathrm{Ub}(\mathrm{n})$-Dendra, bound to this Cdc48 complex in ADP (first incubation), was released after

390 incubation with TEV protease (Figure 6C; lanes 5 and 6 versus lanes 3 and 4), confirming that

391 the UT3 domain is required for the interaction. When the first incubation was performed in ATP

392 to induce Dendra unfolding (Figure S5B), removal of the UT3 domain also caused Ub(n)-Dendra

393 to dissociate from the Cdc48 complex (Figure 6C; lane 10-12 versus 7-9), consistent with the 
idea that unfolded $\mathrm{Ub}(\mathrm{n})$-Dendra was released from the Cdc48 complex after translocation and re-associated with it as in the initiation state (scenario b).

397 To further exclude scenario a, we used a polyubiquitinated fusion protein containing both sfGFP 398 and photoconverted Dendra ((Ub(n)-sfGFP-Dendra) (Figure 6D). Photoconvered Dendra, consisting of two non-covalently associated fragments, is irreversibly unfolded by Cdc48 (Figure S5C), while the single-polypeptide domain of sfGFP can rapidly refold after its translocation (Figure S5D). If a polypeptide segment spanned the pore (scenario a), the refolded, bulky sfGFP 402 domain would prevent backsliding of the substrate through the central pore, and the Cdc48 403 pore loops would interact with the polypeptide segment located inside the central pore. In this 404 case, polyubiquitinated substrate would remain associated with the Cdc48 complex after UT3 removal. However, the results show that the association of Ub(n)-sfGFP-Dendra with Cdc48/UN was drastically reduced whenever the UT3 domain was cleaved off (Figure 6D), regardless of whether Dendra was folded or unfolded (ADP or ATP in the first incubation). Because dissociation after TEV protease cleavage occurred even when the second incubation was performed in ADP (Figure 6D, lane 10), translocation is not required for substrate dissociation

410 following UT3 removal.

To further test whether folded and unfolded polyubiquitinated substrates are bound to the

413 Cdc48 complex in the same way, we performed competition experiments (Figure 6E). Dy800-

414 labeled Ub(n)-Dendra was incubated with Cdc48 complex in either ADP or ATP to generate 415 initiation and post-translocation complexes, respectively (Figure $6 \mathrm{E} ; 1^{\text {st }}$ incubation). As 416 expected, unfolding was only observed in ATP (Figure S5E). Complexes of Dy800-labeled

417 substrate and Cdc48 complex were then isolated and incubated with an excess of Dy680418 labeled $\mathrm{Ub}(\mathrm{n})$-Dendra, again in the presence of ADP or ATP (Figure 6E, $2^{\text {nd }}$ incubation).

419 Regardless of the conditions, the second substrate competed efficiently with the first.

420 Competition in the presence of ADP confirms that the initial binding reaction, including the 421 unfolding of the initiator ubiquitin, is reversible. With ATP in the second incubation, the 422 competitor substrate was also unfolded (Figure 6F). These results show that polyubiquitinated 
423 substrate is bound to the Cdc48 complex in the same way before and after unfolding, implying

424 that substrate can undergo multiple rounds of translocation. Indeed, by varying the

425 concentrations of Cdc48 and substrate, we found that each Cdc48 hexamer can unfold more

426 than one substrate molecule (Figure S5F).

428 Our conclusions are further supported by hydrogen/deuterium exchange (HDX) MS

429 experiments (Figure S6A-C). Cdc48 complex was incubated with photoconverted Ub(n)-Dendra

430 in ADP or ATP, and the samples were subjected to HDX for different time periods and analyzed

431 by MS. All peptides derived from Cdc48, Npl4, and Ufd1 showed only small deuteration

432 differences between ADP and ATP (Figure S6A-C), consistent with the idea that all components

433 of the Cdc48 complex are in the same state before and after substrate unfolding.

435 Additional evidence for this conclusion was obtained by site-specific photocrosslinking followed

436 by MS. Polyubiquitinated substrate was incubated in the presence of ADP or ATP with Cdc48

437 complex containing a photoreactive Bpa probe in the D2 ring (position 602) to generate Cdc48

438 complex with associated folded or unfolded substrate (Figure S7A). The samples were then

439 irradiated, and the crosslinked products (Figure S7B) analyzed by trypsin digestion, followed by

440 nano-liquid chromatography and MS (Figure S7C; S7D). In the presence of ADP, the Bpa probe

441 crosslinked to residues Met $^{1}$ or Gln$^{2}$ of ubiquitin (Figure S7C), in agreement with previous

442 results for the initiation state (Twomey et al., 2019). Similar results were obtained in the

443 presence of ATP when essentially all substrate molecules were unfolded (Figure S7D). No other

444 crosslinked substrate or ubiquitin peptide could be detected, probably because the dwell time

445 of the N-terminal segment of the initiator ubiquitin in the central pore is much longer than that

446 of any other segment.

447

\section{Refolding of translocated ubiquitin molecules}

449 Because the pore of Cdc48 ATPase is rather narrow, all ubiquitin molecules must be in an 450 extended conformation during translocation. To test whether these molecules refold after 451 release from $\mathrm{Cdc} 48$, we again used modification of $\mathrm{Ub}^{13 \mathrm{C}}$ by a maleimide-conjugated fluorescent 
452 dye (see Figure 2E). Cysteine-lacking substrate was polyubiquitinated with $\mathrm{Ub}^{13 \mathrm{C}}$ and incubated

453 in the presence of ADP or ATP with Cdc48 complex containing Ufd1 with a TEV protease

454 cleavage site following the UT3 domain (Ufd1 $\left.{ }^{\mathrm{TEV}}\right)$. Without TEV cleavage, strong ubiquitin

455 modification was observed when all components of the Cdc48 complex were present (Figure

456 7A; lanes 4 and 5 versus 1-3). Importantly, no increase in modification was observed in ATP

457 (lane 4 versus 5), although essentially the entire substrate population was unfolded (Figure

458 S7E). Thus, all ubiquitin molecules positioned between the initiator and substrate, which were

459 unfolded when they passed through the central pore, must have refolded after translocation.

460 When polyubiquitinated substrate was released from the Cdc48 complex by TEV cleavage of

$461 U f d 1^{\operatorname{TEV}}$ (see Figure $6 \mathrm{C}$ ), modification of $\mathrm{Ub}^{13 \mathrm{C}}$ remained the ground level (Figure 7A; lanes 6-

462 10). This result and the similar levels of modification in ADP and ATP indicate that all proximal

463 ubiquitin molecules refold after their translocation through the pore; the polyubiquitinated

464 unfolded substrate rebinds to the Cdc48 complex, with an initiator ubiquitin molecule in the

465 central pore. This conclusion is consistent with our observation that ubiquitin molecules

466 undergo HDX with the same kinetics before and after substrate unfolding (Figure 2C).

\section{DISCUSSION}

Here we have determined the molecular mechanism by which the Cdc48 ATPase complex

471 processes its polyubiquitinated substrates. Our results lead to a model (Figure 7B) that explains

472 how Cdc48 and its mammalian ortholog p97/VCP cooperate with the UN cofactor to

473 disassemble protein complexes and extract proteins from membranes.

475 The ATPase complex first binds a polyubiquitinated substrate, a process that is reversible and

476 requires no ATP hydrolysis (Figure 7B, stage 1 to stage 2). All three components, i.e. Cdc48,

$477 \mathrm{Npl}$, and Ufd1, cooperate in the initial interaction with the ubiquitin chain, which results in the

478 unfolding of one of the ubiquitin molecules. Since all ubiquitin molecules undergo HDX, it

479 seems that the choice of the initiator ubiquitin is random. However, this ubiquitin molecule

480 needs to be succeeded in the chain by several folded ubiquitin molecules, two of which are 
481 bound to the top of the Npl4 tower and two or more to the UT3 domain of Ufd1. Our results

482 indicate that the UT3 interaction is more important. Despite ubiquitin being a very stable

483 protein, it is unfolded simply by binding to the Cdc48 complex. Unfolding is initiated by thermal

484 fluctuation, which results in the separation of the $\mathrm{N}$-terminus of ubiquitin from the rest of the

485 molecule (Irbäck et al., 2005). Next, a ubiquitin segment binds to a groove of Npl4 and the N-

486 terminus is captured by its insertion into the D1 ring of Cdc48. Together, these interactions shift

487 the equilibrium towards the unfolded state of ubiquitin.

During the binding step, the $\mathrm{N}$-terminal segment of the initiator ubiquitin engages the D2 pore loops, as visualized in our previous cryo-EM structure (Twomey et al., 2019). We now show that subsequent ATP hydrolysis causes this segment to be pulled through the central pore

(Figure 7B, stage 2 to 3). As a consequence, the segment of the initiator ubiquitin originally bound to the Npl4 groove is dislodged, which in turn leads to the release of the folded ubiquitin molecules Ub1 and Ub2 from their original binding sites at the top of Npl4. The UT3 domain of Ufd1 probably continues to reversibly interact with the distal parts of the ubiquitin chain.

The next important event happens when the type I branchpoint of the initiator ubiquitin enters the D1 pore (Figure 7B, stage 4). We demonstrate that the ATPase continues translocation towards the C-terminus of the initiator ubiquitin (stage 4 to 5), rather than translocating distal ubiquitin molecules. The selectivity may be determined by the fact that the C-terminal segment of the initiator ubiquitin is already unfolded and therefore requires less energy for translocation than the folded, K48-attached Ub1 molecule. This Ub1 molecule and all ubiquitins distal to it remain folded and outside the central pore. Because the K48 branchpoint of the initiator ubiquitin is translocated through the pore, distal ubiquitins likely move on the side of the double-ring ATPase (Figure 7B, stages 4-6), with a segment between K48 and the C-terminus of 506 the adjacent ubiquitin molecule passing through lateral openings between two protomers of 507 the hexamers. This movement may require some of the distal ubiquitin molecules to dissociate 508 from the UN complex. Lateral opening of the two hexameric ATPase rings seems possible, as 509 both the D1 and D2 protomers can form open spirals, in which one protomer is invisible in cryo- 
510 EM structures (Twomey et al., 2019). A requirement for lateral ring opening is supported by

511 our observation that a disulfide-crosslinked Cdc48 hexamer can bind, but not translocate, a

512 polyubiquitinated substrate.

514 After translocating the initiator ubiquitin, Cdc48 encounters the next branchpoint (Figure 7B, 515 stage 6), as the C-terminus of the initiator is linked to either a lysine residue of the substrate or 516 K48 of another ubiquitin molecule. This type II branchpoint is fundamentally different from the 517 one in the initiator ubiquitin (see scheme in Figure 5A). In this case, Cdc48 can translocate both 518 polypeptide branches through the pore, as demonstrated by the unfolding of fluorescent 519 substrates located at either the $\mathrm{N}$ - or C-terminus of fusion proteins. Because Cdc48 translocates 520 sequentially all ubiquitin molecules positioned between the initiator and substrate (proximal 521 ubiquitins), it encounters many type II branchpoints during substrate processing, and at each of 522 them likely unfolds both $\mathrm{N}$ - and $\mathrm{C}$-terminal segments. The proximal ubiquitin molecules are all 523 transiently unfolded but refold after translocation, while most substrates remain unfolded

524 (Figure 7B, stage 7 to 8 ).

526 Because the D2 pore loops of Cdc48 surround a single polypeptide chain, it is possible that the 527 ATPase translocates only one polypeptide branch at any given time and needs multiple rounds 528 to unfold the entire substrate population. However, such a mechanism seems rather wasteful. 529 An alternative is that Cdc48 moves several polypeptide chains simultaneously through its pore. 530 Indeed, a structure of the related Vps4 ATPase with a circular peptide substrate indicates that a 531 second strand can be accommodated (Han et al., 2019), and single-molecule experiments

532 with the ClpB ATPase suggest that two strands can be processed at the same time (Avellaneda 533 et al., 2020).

535 At the end of translocation, the entire polypeptide is released from the ATPase complex, 536 because proximal ubiquitins and substrate translocate through the central pore, and distal 537 ubiquitins move outside the pore (Figure 7B; stage 7). However, the unfolded, 538 polyubiquitinated can re-bind to the ATPase complex, re-establish an initiation complex (similar 
539 to stage 2 in Figure 7B; not shown), and begin a new translocation cycle. The start of

540 translocation seems to be rate-limiting as, according to our photocrosslinking experiments, the

541 initiation state is most populated. For efficient transfer of the unfolded, polyubiquitinated

542 substrate to the proteasome, the ubiquitin chain needs to be shortened by a deubiquitinase

543 (DUB) to weaken its affinity for the ATPase complex. Indeed, Otu1, a DUB that binds to the $\mathrm{N}$

544 domains of Cdc48, can trim the ubiquitin chain (Bodnar and Rapoport, 2017b).

Our proteomics results indicate that the Cdc48 ATPase complex acts in vivo on the majority of

547 polyubiquitinated proteins, showing little specificity for the actual substrate. This conclusion is

548 consistent with our in vitro experiments and cryo-EM structure, which both show that the

549 Cdc48 complex only recognizes ubiquitin molecules. Cofactors other than Ufd1 and Npl4, such

550 as UBA-UBX proteins interacting with both Cdc48 and ubiquitin (Hänzelmann and Schindelin,

551 2017), could increase the binding constant for polyubiquitin chains, but since there are only a

552 few of them, they cannot provide much substrate specificity. This raises the question as to how

553 the Cdc48 complex, the proteasome, and the shuttling factors Rad23 and Dsk2 (Finley et al.,

554 2012) divide their tasks, as they all seem to primarily recognize the ubiquitin chain, with little

555 or no specificity for the actual substrate. In addition, the Cdc48/UN complex and the 26S

556 proteasome require ubiquitin chains of about the same length (5 versus 4 ubiquitins (Bodnar

557 and Rapoport, 2017b; Thrower et al., 2000)). So far, it has been assumed that Cdc48 is the

558 most upstream component, then delivers substrates to the shuttling factors, which in turn bring

559 them to the proteasome for degradation. However, with our new data, one would have to

560 assume that Cdc48 unfolds every protein that carries a sufficiently long ubiquitin chain, a

561 scenario that seems rather wasteful. An alternative is that the proteasome has the first pick

562 (Figure 7C); only if a polyubiquitinated protein cannot be degraded would Cdc48 have a chance.

563 In this model, the proteasome would act both upstream and downstream of the Cdc48

564 complex. Shuttling factors would transfer polyubiquitinated proteins back and forth between

565 the Cdc48 complex and the proteasome, regardless of whether they are folded or unfolded

566 (Figure 7C). They would pick up these proteins from the initiation state of the Cdc48 ATPase,

567 the most populated state during substrate processing. Transfer between the Cdc48 complex 
568 and the $26 \mathrm{~S}$ proteasome would be facilitated by DUB-mediated shortening of the ubiquitin

569 chain. This model can now be tested with reconstituted systems containing purified Cdc48

570 complex, 26S proteasomes, shuttling factors, and possibly other Cdc48-interacting proteins.

571

572 


\section{ACKNOWLEDGEMENTS}

574 We thank the ICCB-Longwood Screening Facility for use of equipment. We thank Johannes

575 Walter, Nicholas Bodnar, and Olga Kochenova for critical reading of the manuscript. This work

576 was supported by a NIGMS grant (R01 GM052586) to T.A.R., by a research collaboration

577 between J.R.E. and the Waters Corporation, by NIH grants (R01 CA233800 and R21 CA247671)

578 to J.A.M., by financial support to J.A.M. from the Dana-Farber \& Northeastern University Joint

579 Program in Cancer Research, by an NIH/NIGMS grant (R01 GM132129) to J.A.P., and by an

580 NIGMS grant (R01 GM067945) to S.P.G. Z.J. is a Howard Hughes Medical Institute Fellow of the

581 Damon Runyon Cancer Research Foundation, DRG-2315-18. T.A.R. is a Howard Hughes Medical

582 Institute Investigator.

583

584 AUTHOR CONTRIBUTION

585 Z.J. and H.L. performed protein purifications, protein labeling, and all unfolding, binding, and 586 photocrosslinking assays. D.P., T.E.W., and J.R.E. conducted the HDX MS analyses, J.A.P. and

587 S.P.G. performed TMT labeling and MS analysis, and S.B.F. and J.A.M. analyzed crosslinked

588 peptides by MS. T.A.R. supervised the project. Z.J. and T.A.R. wrote a draft of the manuscript.

590 DECLARATION OF INTERESTS

591 J.A.M. serves on the SAB of 908 Devices and receives sponsored research support from

592 AstraZeneca and Vertex. All other authors declare no competing interests. 
Figure legends:

Figure 1. Most proteins carrying K48-linked ubiquitin chains interact with the Cdc48/UN

599 (A) S. cerevisiae cells lacking the ABC transporter Pdr5 (BY4741:pdr5 $\Delta$ ) and expressing Npl4-

600 FLAG or Ufd1-FLAG from plasmids were incubated with or without the proteasome inhibitor 601 bortezomib (BTZ). Cell lysates were incubated with either beads containing FLAG antibodies 602 (Anti-FLAG resin) or biotinylated TUBE recognizing K48-linked ubiquitin chains $\left({ }^{B i o} T^{T B E^{K 48}}\right.$ ) and 603 streptavidin beads. Polyubiquitinated proteins were eluted with an excess of trypsin-resistant 604 TUBE (TR-TUBE). The samples were subjected to trypsin digestion, TMT labeling, and analysis by 605 tandem MS.

606 (B) For each substrate protein detected in BTZ-treated cells, its abundance in the FLAG 607 immunoprecipitation (IP) was divided by its abundance in the K48 ubiquitin pull-down (K48 IP) 608 (Cdc48/K48 ratios). The ratios from Npl4-FLAG and Ufd1-FLAG cells were plotted against each 609 other on a logarithmic scale.

610 (C) As in (B), but for untreated cells (DMSO instead of BTZ).

611 (D) As in (B), but comparing data from Npl4-FLAG expressing cells with or without BTZ 612 treatment.

613 (E) As in (D), but for Ufd1-FLAG expressing cells.

614 (F) For each detected protein, the Cdc48/K48 ratios were averaged among all tested cell 615 lysates. Proteins between the dotted lines are enriched or depleted by a factor of less than 616 three.

618 Figure 2. Cdc48-mediated ubiquitin unfolding.

619 (A) Scheme of the Ub(n)-Dendra substrate employed for in vitro experiments.

620 (B) Experimental protocol of the HDX experiments.

621 (C) Photoconverted Ub(n)-Dendra was incubated with or without Cdc48/UN complex in the 622 presence of ADP or ATP. The samples were then subjected to HDX for different time periods, 623 the proteins were subjected to proteolytic cleavage, and the deuteration of ubiquitin peptides 
624 determined by MS. Ubiquitin peptides covered the indicated amino acids. Shown is the

625 difference in the deuterium level (in Daltons) caused by binding of the Cdc48 complex (color 626 scale shown in the left panel).

627 (D) As in (C), but for the Dendra-fusion protein. Peptides covering amino acids 153-192 contain

628 the two N-terminal $\beta$-strands of Dendra unfolded during translocation.

629 (E) Cysteine-free Dendra substrate was polyubiquitinated with ubiquitin carrying a Cys at 630 position 3, resulting in $\mathrm{Ub}^{13 \mathrm{C}}(\mathrm{n})$-Dendra. $\mathrm{Ub}^{13 \mathrm{C}}(\mathrm{n})$-Dendra was then incubated with different 631 combinations of $\mathrm{Npl} 4, \mathrm{Ufd} 1$, and full-length $\mathrm{Cdc} 48\left(\mathrm{Cdc} 48^{\mathrm{FL}}\right)$ or $\mathrm{Cdc} 48$ containing only the $\mathrm{N}$ and

632 D1 domains $\left(\mathrm{Cdc} 48^{\mathrm{ND} 1}\right)$. The samples were incubated with a maleimide-conjugated fluorescent 633 dye (Dy-maleimide) and analyzed by SDS-PAGE, followed by fluorescence scanning (upper 634 panel) and Coomassie-blue staining (lower panel). Ubiquitin modification was quantitated by 635 measuring fluorescence intensities (numbers under the lanes).

636 (F) Ub(n)-Dendra carrying a fluorescent dye was incubated in the presence of ADP with 637 different combinations of Ufd1, Npl4-FLAG, and Cdc48. FLAG antibody beads (Anti-FLAG) were 638 added, and bound material analyzed by SDS-PAGE, followed by fluorescence scanning (upper 639 panel) and Coomassie-blue staining (lower panel). To evaluate the pull-down efficiency, 640 different amounts of the input material were loaded (left four lanes).

643 ring.

644 (A) Cdc48 lacking the D2 domain (Cdc48 $\left.{ }^{\mathrm{ND} 1}\right)$, which was otherwise wild-type (WT), lacked the 645 pore loops in the D1 domain ( $\triangle \mathrm{D} 1$ loops), or was deficient in ATPase activity (E315A), was 646 incubated with SBP-tagged Ufd1 (SBP-Ufd1), Npl4, and dye-labeled Ub(n)-sfGFP in the presence 647 of ADP. The Cdc48 complex was retrieved with streptavidin beads, and bound material analyzed 648 by SDS-PAGE, followed by fluorescence scanning (upper panel) and Coomassie-blue staining 649 (lower panel). Substrate was quantitated by measuring fluorescence intensities (numbers under 650 the lanes).

651 (B) Photoconverted Ub(n)-Dendra was incubated in the presence of ATP with Cdc48/UN 652 complex containing full-length wild-type (WT) Cdc48, or the D1 mutants $\triangle \mathrm{D} 1$ loops or E315A. 
653 Unfolding of Dendra was followed by the loss of fluorescence. The experiments were

654 performed in triplicates. Shown are means and standard deviations for each data point.

655 (C) Scheme for testing whether the N-terminus of the initiator ubiquitin enters the D1 ring from

656 the side. Lateral entry is prevented by crosslinking all six D1 domains in the hexameric ring

657 through disulfide bridges positioned close to the entrance of the pore.

658 (D) Crosslinked Cdc48 hexamers were generated with Cdc48 containing S286C and R333C

$659\left(\mathrm{Cdc}^{2 \mathrm{Cys}}\right)$ by addition of an oxidant (oxidized). One aliquot was treated with a reducing

660 reagent to generate non-crosslinked hexamers (reduced). The samples were incubated with

661 Ufd1, Npl4-FLAG, and photoconverted dye-labeled Ub(n)-Dendra in the presence of ADP,

662 followed by retrieval of the Cdc48 complex with FLAG-antibody beads. As a control, wild-type

663 Cdc48 was used. Bound material was analyzed by reducing and non-reducing SDS-PAGE,

664 followed by fluorescence scanning and Coomassie-blue staining.

665 (E) Cdc48/UN complex containing wild-type Cdc48 (Cdc48 $\left.{ }^{\mathrm{WT}}\right), \mathrm{Cdc} 48^{2 \mathrm{Cys}}$ (oxidized), or Cdc48 ${ }^{2 \mathrm{Cys}}$

666 (reduced) was tested for unfolding of photoconverted Ub(n)-Dendra, as in (B).

667 (F) The ubiquitin chain in photoconverted Ub(n)-Dendra was replaced with a chain of Dendra-

668 ubiquitin fusions containing a TEV protease cleavage site between the fusion partners ([Dendra-

$669 \mathrm{Ub}](\mathrm{n})$-Dendra; see scheme). Note that Dendra in the fusions was not photoconverted. After

670 incubation with or without TEV protease (TEVp), unfolding of photoconverted Dendra was

671 tested in the absence or presence of Cdc48 complex, as in (B).

672

673 Figure 4. Translocation of the initiator ubiquitin by Cdc48.

674 (A) Model of Npl4 with bound ubiquitin molecules, based on a cryo-EM structure of Cdc48 in

675 complex with polyubiquitinated substrate (PDB, 6OA9). Npl4 is shown in grey, the unfolded

676 initiator ubiquitin molecule in red, the two folded ubiquitin molecules Ub1 and Ub2 in pink, and

677 Cdc48 in blue. Bpa probes were incorporated at the indicated Npl4 positions.

678 (B) The unfolding of photoconverted Ub(n)-Dendra was tested with Cdc48 complex containing

679 wild-type Npl4 (Npl4 ${ }^{\mathrm{WT}}$ ) or Npl4 with Bpa probes at positions interacting with the initiator

680 ubiquitin, Ub1, or Ub2 (Npl $4^{\mathrm{Bpa}}$; the curve colors correspond to the positions shown in $(\mathrm{A})$ ) with

681 (w) or without (w/o) UV-induced crosslinking. 
682 (C) For each Bpa mutant, the percentage of substrate crosslinked to Npl4 was determined

683 (Figure S4B) and compared with the percentage of inhibition of the initial rate of Dendra

684 unfolding.

685

Figure 5. Translocation of branched polypeptides by the Cdc48 ATPase.

(A) Scheme of substrate processing by the Cdc48 ATPase complex. Ubiquitin molecules proximal and distal from the initiator (in red) are indicated in pink and purple, respectively. The two different branchpoints (type I and II) are indicated by dashed boxes in the upper scheme and magnified in the lower panels. Arrows indicate the directions of translocation through the Cdc48 pore.

692 (B) Schemes of different polyubiquitinated substrates used for unfolding experiments. Proteins 693 linked by fusion are shown in a dashed box, with $\mathrm{N}$ - and C-terminus indicated. Dist and Prox 694 indicate that photoconverted Dendra is located either at the distal or proximal end of a 695 polyubiquitin chain. $(\mathrm{N})$ and $(\mathrm{C})$ indicate that Dendra is located at the $\mathrm{N}$ - or $\mathrm{C}$ - terminus of the 696 fused ubiquitin.

697 (C) The substrates shown in (B) were tested for unfolding of photoconverted Dendra by measuring the loss of fluorescence. The experiments were performed in triplicates. Shown are 699 means and standard deviations for each data point.

700 (D) A polyubiquitinated substrate was generated with a fusion protein containing 701 photoconverted Dendra, ubiquitin, and sfGFP (dashed box) and tested for the unfolding of 702 Dendra.

703 (E) As in (D), but with swapped positions of sfGFP and Dendra in the fusion protein.

Figure 6. Release of polyubiquitinated substrate from the Cdc48 ATPase complex.

706

(A) Photoconverted Ub(n)-Dendra, Cdc48, and Ufd1 were incubated with or without FLAG-

707 tagged Npl4 in the presence of ADP or ATP. The Cdc48 complex was isolated with FLAG-

708 antibody beads, and bead-bound material analyzed by SDS-PAGE, followed by immunoblotting (IB) with ubiquitin antibodies (anti-Ub; upper panel) and Coomassie-blue staining (lower panel). 
710 (B) Scheme showing two conceivable scenarios for the post-translocation state. In scenario (a),

711 the distal ubiquitins (in purple) remain on the cis side of the ATPase ring, resulting in a

712 polypeptide segment spanning the pore (dashed line). In this case, the substrate cannot be

713 released by removal of the UN cofactor. In scenario (b), distal ubiquitin molecules move outside

714 the ATPase rings, while the K48 branchpoint of the initiator ubiquitin (in red) moves through

715 the pore. At the end, the unfolded, polyubiquitinated substrate is released from Cdc48 and can

716 rebind to the ATPase complex.

717 (C) Dye-labeled Ub(n)-Dendra was incubated in the presence of ADP or ATP with Cdc48, Npl4-

718 FLAG, and Ufd1 containing a TEV cleavage site following the UT3 domain (Ufd1 $\left.1^{\text {TEV }}\right)\left(1^{\text {st }}\right.$

719 incubation). The Cdc48 complex was retrieved with FLAG-antibody beads, and bound material

720 was treated with TEV protease in the presence of ADP, ATP, or ATP $\gamma$ S, a non-hydrolyzable ATP

721 analog ( $2^{\text {nd }}$ incubation). Bead-bound material was then analyzed by SDS-PAGE, followed by

722 fluorescence scanning (upper panel) and Coomassie-blue staining (lower panel).

723 (D) As in (C), but with Ub(n)-sfGFP-Dendra. Note that sfGFP refolds after translocation, which

724 would prevent backsliding of the polypeptide through the Cdc48 pore in scenario (a) (upper

725 panel).

726 (E) Photoconverted Ub(n)-Dendra labeled with the fluorophore DyLight800 (Ub(n)-Dy800-

727 Dendra) was incubated with Cdc48-FLAG, SBP-Ufd1, and Npl4 in the presence of ADP or ATP (1 ${ }^{\text {st }}$

728 incubation). The Cdc48 complex was retrieved with streptavidin beads and eluted with biotin.

729 The samples were then incubated with DyLight680-labeled, photoconverted Ub(n)-Dendra

$730(\mathrm{Ub}(\mathrm{n})$-Dy680-Dendra) in the presence of ADP or ATP. Anti-FLAG antibody beads were added,

731 and bound material analyzed by SDS-PAGE, followed by fluorescence scanning at 800nm and

$732680 \mathrm{~nm}$ wavelength (upper two panels) and Coomassie-blue staining (lower panel).

733 (F) Samples in (E), incubated in ADP or ATP during the first incubation, were tested for

734 unfolding of photoconverted Ub(n)-Dy680-Dendra in ATP during the second incubation (Figure

$7356 \mathrm{E}$; lanes 9 versus 12$)$.

736

737 Figure 7. Refolding of translocated ubiquitin and model for substrate processing by the Cdc48

738 complex. 
739 (A) $\mathrm{Ub}^{13 C}(\mathrm{n})$-Dendra was incubated in the presence of ADP or ATP with Cdc48-FLAG, Npl4-FLAG, 740 and HA-tagged Ufd1 containing a TEV protease cleavage site following the UT3 domain (HA-

$\left.741 \mathrm{Ufd} 1^{\mathrm{TEV}}\right)$. Where indicated, TEV protease (TEVp) was added after incubation with the

742 nucleotides. All samples were then incubated with a maleimide-conjugated fluorescent dye (Dy-

743 maleimide) and analyzed by SDS-PAGE, followed by fluorescence scanning (upper panel),

744 Coomassie-blue staining ( $2^{\text {nd }}$ panel), and immunoblotting (IB) with ubiquitin, FLAG, and HA

745 antibodies. The HA antibodies cross-react with TEV protease, which migrates at the same

746 position as UT3 (star).

747 (B) Scheme of substrate processing by the Cdc48 ATPase complex. The boxed initiation state is 748 most populated. The unfolded initiator ubiquitin is shown as a red line, and proximal and distal 749 ubiquitin molecules as pink and purple circles, respectively. Translocated, unfolded ubiquitin 750 and substrate molecules are indicated as spirals. Unfolded substrate released from the Cdc48 751 complex can be transferred by shuttling factors to the 265 proteasome and be degraded.

752 (C) Model for the transfer of polyubiquitinated proteins between the Cdc48 complex and the 753265 proteasome. Note that both particles and the shuttling factors all recognize primarily the 754 ubiquitin chain. 
STAR * METHODS

759

760

RESOURCE AVAILABILITY

761

Lead Contact

762 Further information and requests for resources and reagents should be directed to and will be

763 fulfilled by the Lead Contact, Tom Rapoport (tom rapoport@hms.harvard.edu).

Materials Availability

766 All unique/stable reagents generated in this study are available from the Lead Contact with a

767 completed Materials Transfer Agreement.

Data and Code Availability

770 The HDX MS data have been deposited to the ProteomeXchange Consortium via the PRIDE

771 partner repository with the dataset identifier PXD027639.

772

773 EXPERIMENTAL MODEL AND SUBJECT DETAILS

774 Yeast strains and cultures

775 Plasmids encoding Saccharomyces cerevisiae Uba1 and Ubr1 were transformed into the INVSc1

776 yeast strain (Thermo). The yeast cells were grown in synthetic dropout (SD) medium for $24 \mathrm{~h}$,

777 and then switched to yeast culturing medium containing $2 \%$ galactose to induce protein

778 expression. The cells were harvested after $24 \mathrm{~h}$ of the galactose induction.

780 The $p d r 5 \Delta$ strain was derived from BY4741 as described in (Yip et al., 2020). The pRS413(His)

781 plasmid encoding either Npl4-FLAG or Ufd1-FLAG was transformed into the pdr5 $\Delta$ strain.

782 Positive clones on SD-Leu-His plates were pooled for subsequent experiments. Overnight

783 cultures of yeast expressing either Npl4-FLAG or Ufd1-FLAG were inoculated into 80 ml of SD-

784 Leu-His medium at an $\mathrm{OD}_{600}$ of 0.1 . Cells were grown at $30^{\circ} \mathrm{C}$ until an $\mathrm{OD}_{600}$ of 0.7 . The cells

785 were then treated with $80 \mu \mathrm{M}$ bortezomib (Selleckchem) or the same volume of DMSO and 
incubated at $30^{\circ} \mathrm{C}$ for another 4 h. $120 \mathrm{OD}_{600}$ units of cells were spun down, and flash-frozen in

787 liquid nitrogen.

\section{Bacteria cultures}

790 Bacterial expressing plasmids were transformed into Escherichia coli BL21 CodonPlus (DE3) RIPL 791 cells (Agilent), unless stated otherwise. Bacterial strains were grown in Terrific Broth to an

$792 \mathrm{OD}_{600}$ of 0.8 . Protein expression was induced by addition of $0.1 \mathrm{mM}$ isopropyl b-D-1-

793 thiogalactopyranoside (IPTG), and then the incubation was continued at $16^{\circ} \mathrm{C}$ for $16 \mathrm{~h}$.

795 p-Benzoyl-phenylalanine (Bpa) was incorporated into proteins by amber codon suppression in 796 Escherichia coli BL21 (DE3) (New England BioLabs) harboring the plasmid pEVOL-pBpF(Chin et 797 al., 2002). Cells were grown in Terrific Broth to an $\mathrm{OD}_{600}$ of 0.8. Protein expression was induced 798 by the addition of $0.02 \%$ L-arabinose, $1 \mathrm{mM} \mathrm{Bpa,} \mathrm{and} 0.2 \mathrm{mM}$ IPTG, and the incubation was 799 continued at $16^{\circ} \mathrm{C}$ for $16 \mathrm{~h}$.

\section{METHOD DETAILS}

\section{Plasmids}

803 For yeast experiments, the endogenous loci of the Saccharomyces cerevisiae np/4 and ufd1 804 genes - including their promoters, coding regions, and terminators - were amplified by 805 polymerase chain reaction (PCR) and cloned into a yeast centromeric vector, pRS413(His3), 806 using the Notl and Xhol restriction enzyme sites. A sequence encoding the FLAG tag 807 (DYKDDDDK) was inserted at the C-terminus of each gene to express FLAG tagged Npl4 and 808 Ufd1. S. cerevisiae uba1 was cloned into the pRS426Gal1 vector with a His14-tag 809 (HHHHSGHHHTGHHHHSGSHHH) and a TEV-protease cleavage site (ENLYFQG), as described in 810 (Stein et al., 2014). S. cerevisiae ubr1 gene was also cloned into pRS426Gal1(His14-TEV), using 811 Notl and Ascl sites, resulting the N-terminal sequence MSKHHHHSGHHHTGHHHHSGSHHHG812 ENLYFQ-GAAA. 
814 Wild-type Cdc48 and its variants were cloned into the pET28 vector using Notl and Ascl sites,

815 with a His6-tag and a TEV-protease cleavage site at the N-terminus. A sequence encoding the

816 FLAG tag was added at the C-terminus of $\mathrm{Cdc} 48$, as appropriate. $\mathrm{Cdc} 48^{\mathrm{ND} 1}$ contains residues 1-

817480 of wild-type Cdc48. Cdc48 4 D1Loops contains internal deletions of residues 286-290 and

818 residues $325-330$ of wild-type Cdc48. All Ufd1 variants were cloned into the pK27 vector with

819 an N-terminal His14-SUMO (small ubiquitin-like modifier) tag. A sequence encoding the

820 hemagglutinin (HA)-tag (YPYDVPDYA), or a streptavidin-binding protein (SBP) tag was inserted

821 between SUMO and Ufd1, where indicated. Ufd1 $U$ UT3 contains a truncation of the first 200

822 residues of Ufd1. Ufd1 $1^{\text {TEV }}$ has inserted a TEV-protease cleavage site (ENLYFQG) between the

823 residues 209 and 210 of Ufd1. All Npl4 variants were cloned into the pET21 vector using Ndel

824 and Ascl sites, with a C-terminal His6-tag, FLAG tag, or FLAG-His6 tag. Internal deletions and

825 insertions as well as point mutations were generated by overlapping PCR.

827 Wild-type human ubiquitin (hUb) and the I3C mutant were cloned into the pK27(His14-SUMO)

828 vector using the Gibson assembly method. A sequence encoding a single Ala residue was

829 inserted between the SUMO tag and the ubiquitin gene to allow for cleavage of the SUMO tag

830 by Ulp1. All N-end rule degron fusions with a fluorescent substrate were constructed by

831 overlapping PCR, and then cloned into the pK27(His14-SUMO) vector. The fluorescent

832 substrates used in this study include the cysteine-free moxDendra2 (Dendra), the lysine-less

833 super-folder GFP (sfGFP; a gift from Dirk Goerlich), the sfGFP-GGGSGGGSGGGS-Dendra fusion,

834 and mEos3.2 (Eos; as described in (Bodnar and Rapoport, 2017b)). The sequence of the N-

835 end rule degron is as follows (note that an N-terminal arginine is generated after SUMO

836 cleavage): RHGSGCGAWLLPVSLVKRKTTLAPNTQTASPPSYRALADSLMQ. For substrates used in

837 ubiquitin modification by a maleimide-conjugated fluorescent dye, the cysteine in the $\mathrm{N}$-end

838 rule degron was mutated to serine. All ubiquitin fusions with fluorescent proteins were

839 constructed by overlapping PCR and cloned into the pET28(His6-FLAG) vector using the BamHI

840 and $\mathrm{Ascl}$ sites.

841 The bacterial expression plasmid encoding S. cerevisiae Ubc2 has been described in (Bodnar

842 and Rapoport, 2017b). Plasmid encoding mouse Ube1 was a gift from Jorge Eduardo Azevedo 
843 (Addgene plasmid \# 32534). The coding region of gp78 ${ }^{\mathrm{RING}}-\mathrm{Ube} 2 \mathrm{~g} 2$ was constructed as

844 described previously (Blythe et al., 2017) and cloned into the pET28(His6-TEV) vector using 845 Notl and Ascl sites. The gp78 ${ }^{\text {RING }}$-Ube2g2 fusion protein contains the RING domain of human 846 gp78 (residue 322-393) and human Ube2g2 with the linker sequence GTGSH in between. The 847 gene for human gp78 was a gift from Allan Weissman (Addgene plasmid \# 37375). The gene for 848 human Ube2g2 was a gift from Wade Harper (Addgene plasmid \# 15791). The trypsin-resistant 849 tandem ubiquitin binding entity (TR-TUBE) was cloned into pET28 vector using the Notl and Ascl 850 sites, resulting in the N-terminal sequence MGHHHHHHGSGENLYFQGAAACDI. The gene for TR851 TUBE was a gift from Yasushi Saeki (Addgene plasmid \# 110313). The pEVOL-pBpF plasmid used 852 to produce Bpa-incorporated proteins was a gift from Peter Schultz (Addgene plasmid \# 31190).

\section{Immunoblotting and antibodies}

855 Antibodies used in this study were: anti-Cdc48 (MyBioSource, MBS423348, 1:500), anti-

856 ubiquitin (Santa Cruz Biotechnology, clone P4D1, 1:200), anti-FLAG (Sigma, clone M2, 1:1000),

857 anti-HA (Roche, clone 12CA5, 1:1000), anti-ubiquitin K48-specific (Cell Signaling Technology,

858 clone D9D5, 1:1000), anti-PGK1 (Abcam, clone 22C5D8, 1:1000), anti-SBP-tag (Millipore, clone

859 20, 1:1000), donkey anti-mouse IgG DyLight 800 conjugated (ThermoFisher, 1:5000), donkey

860 anti-mouse IgG DyLight 680 conjugated (ThermoFisher, 1:5000), donkey anti-rabbit IgG DyLight

861800 conjugated (ThermoFisher, 1:5000), donkey anti-goat IgG H\&L horseradish peroxidase

862 (HRP)-conjugated (Abcam, ab97110, 1:5000). The substrate for HRP conjugated secondary

863 antibodies was Western Lighting Ultra (Perkin Elmer, NEL111001EA).

\section{Protein purifications}

866 All purified proteins were snap-frozen in size-exclusion chromatography (SEC) buffer (50 mM 867 HEPES, pH 7.4, $150 \mathrm{mM} \mathrm{NaCl}, 5 \mathrm{mM} \mathrm{MgCl}_{2}$, and $0.5 \mathrm{mM}$ tris(2-carboxyethyl)phosphine (TCEP)), 868 except Cdc48 2 cys , for which TCEP was omitted.

$870 \mathrm{Cdc} 48$, untagged Ufd1/Npl4 (UN), and the UN complexes harboring the groove mutants of Npl4 871 were expressed and purified as previously described (Twomey et al., 2019). Bacterial cells 
872 expressing Cdc48 2 Cys were harvested by centrifugation at $5000 \mathrm{xg}$ for $10 \mathrm{~min}$ and resuspended

873 in wash buffer (50 mM Tris- $\mathrm{HCl}$, pH 8, $320 \mathrm{mM} \mathrm{NaCl}, 5 \mathrm{mM} \mathrm{MgCl}$, $10 \mathrm{mM}$ imidazole, $0.5 \mathrm{mM}$

874 ATP) supplemented with phenylmethylsulfonyl fluoride (PMSF; 1 mM), a protease inhibitor

875 cocktail, and DNase I (5 $\mathrm{gg} / \mathrm{ml})$. The cells were lysed by sonication. Lysates were cleared by

876 ultracentrifugation in a Ti-45 rotor (Beckman) at $40,000 \mathrm{rpm}$ for $30 \mathrm{~min}$ at $4^{\circ} \mathrm{C}$. The

877 supernatants were incubated with Ni-NTA resin that was pre-equilibrated with wash buffer, for

$87860 \mathrm{~min}$ at $4^{\circ} \mathrm{C}$. The resin was washed three times with 30 column volumes of wash buffer.

879 Proteins were eluted with elution buffer $(50 \mathrm{mM}$ Tris- $\mathrm{HCl}$, pH 8, $150 \mathrm{mM} \mathrm{NaCl}, 5 \mathrm{mM} \mathrm{MgCl}$, $880400 \mathrm{mM}$ imidazole), and the eluates were diluted to about $800 \mathrm{nM}$ and treated with $10 \mu \mathrm{M}$ of 881 the oxidant 4,4'-dipyridyl disulfide (Sigma) at $30^{\circ} \mathrm{C}$ for $30 \mathrm{~min}$. After incubation, the reaction 882 mixture was dialyzed against $50 \mathrm{mM}$ HEPES, pH 7.5, $150 \mathrm{mM} \mathrm{NaCl}, 5 \mathrm{mM} \mathrm{MgCl} 2$ at $4^{\circ} \mathrm{C}$ 883 overnight before snap-freezing.

Individual Ufd1 proteins were purified by Ni-NTA resin as described above. The SUMO protease

886 Ulp1 was added to the eluted Ufd1 protein and dialyzed against wash buffer containing $10 \mathrm{mM}$ 887 imidazole. The Ulp1-treated samples were incubated with Ni-NTA resin to remove the His14-

888 SUMO tag, and the unbound proteins were concentrated and loaded onto a Superdex 200 889 Increase column equilibrated with SEC buffer. Npl4 proteins were purified similarly by Ni-NTA 890 and Superdex 200 Increase chromatography. Proteins with incorporated Bpa were purified in 891 the same way as their parental counterparts.

893 Fluorescent substrates (Dendra, sfGFP, or Eos) containing the N-end rule degron and His14-

894 SUMO tag were purified by Ni-NTA resin followed by the SUMO tag cleavage, SUMO tag 895 removal with Ni-NTA resin, and gel filtration, similarly to the purification of the Ufd1 protein. 896 Fluorescent substrates with ubiquitin fusions were purified similarly to the Npl4 protein by $\mathrm{Ni}$ 897 NTA resin and gel filtration.

899 His14-Uba1 was expressed in yeast cells and purified by Ni-NTA, ion-exchange, and size900 exclusion chromatography, as described in (Stein et al., 2014). His14-Ubr1 was expressed and 
901 purified by Ni-NTA in the same way as His14-Uba1. After elution from the Ni-NTA resin, the

902 protein was then buffer-exchanged into SEC buffer, concentrated, and snap-frozen. Ubc2 was

903 expressed and purified as previously described (Twomey et al., 2019). Mus musculus Ube1,

904 the gp78 ${ }^{\text {ING }}$-Ube2g2 fusion, His14-SUMO-hUb, His14-SUMO-hUb ${ }^{13 C}$ and TR-TUBE were purified

905 by Ni-NTA and size-exclusion chromatography. An additional step of SUMO cleavage and

906 removal was performed prior to the gel filtration to purify wild-type hUb and the I3C mutant.

907 Note that an extra alanine reside was left at the N-terminus of the purified hUb and hUb $\mathrm{b}^{13 \mathrm{C}}$ after

908 SUMO cleavage. S. cerevisiae ubiquitin was purchased from Boston Biochem.

909

Dye labeling of substrates containing the $\mathbf{N}$-end rule degron

911 The purified substrates were reduced with $10 \mathrm{mM}$ TCEP and then incubated with a 3-fold molar

912 excess of maleimide-conjugated DyLight dyes (Thermo). The reactions were kept in the dark at

913 room temperature for $2 \mathrm{~h}$ before quenching with $20 \mathrm{mM}$ dithiothreitol (DTT). The unreacted

914 free dyes were removed by Dye Removal columns (Thermo, \#22858).

Photoconversion of substrates containing Dendra or Eos

917 The purified substrate proteins $(4 \sim 8 \mathrm{mg} / \mathrm{ml})$ were placed in a $200-\mu \mathrm{l}$ PCR tube in an ice bath. A

918 long-wavelength UV flashlight (395-410 $\mathrm{nm}$, DULEX) was positioned $5 \mathrm{~cm}$ above the tube, and

919 the sample was irradiated for $1 \mathrm{~h}$, with occasional mixing.

921 Ubiquitination of substrates

922 Ubiquitination of the substrates containing an $\mathrm{N}$-end rule degron was carried out as previously

923 described (Twomey et al., 2019), with some modifications. Substrate (5 $\mu \mathrm{M})$ was incubated

924 with S. cerevisiae ubiquitin or purified human ubiquitin $(250 \mu \mathrm{M})$, Uba1 (800 nM), Ubc2 (4.63

$925 \mu \mathrm{M})$, Ubr1 $(800 \mathrm{nM})$, and ATP $(10 \mathrm{mM})$ for $60 \mathrm{~min}$ at $30^{\circ} \mathrm{C}$ in ubiquitination buffer $(50 \mathrm{mM}$ Tris

$926 \mathrm{pH}$ 8.0, $150 \mathrm{mM} \mathrm{NaCl}, 10 \mathrm{mM} \mathrm{MgCl}_{2}, 1 \mathrm{mM}$ DTT). The samples were concentrated and loaded

927 onto a Superdex 200 column in SEC buffer. After analysis by SDS-PAGE, fractions containing the

928 desired ubiquitin chain lengths were pooled and snap-frozen. The concentration of the pooled

929 polyubiquitinated substrate was determined with a Synergy Neo2 Multi-mode reader (BioTek), 
using the non-ubiquitinated substrates as standards. The majority of the final product contained polyubiquitin chains of 10-25 ubiquitin molecules. The [Dendra-Ub](n)-Dendra

932 substrate in Figure 3F was generated in a reaction containing $4 \mu \mathrm{M}$ dye-labeled, photoconverted Dendra fusion with N-end rule degron and $50 \mu \mathrm{M}$ Dendra-hUb fusion.

Ubiquitination of the ubiquitin fusion substrates were performed as described in (Blythe et al., 9362017 ) with some modifications. $10 \mu \mathrm{M}$ the ubiquitin-fusion substrate was incubated with $1 \mu \mathrm{M}$ 937 mouse Ube1, $20 \mu \mathrm{M}$ gp78 ${ }^{\text {RING }}$-Ube2g2, and $500 \mu \mathrm{M}$ purified human ubiquitin in $20 \mathrm{mM}$ HEPES, $938 \mathrm{pH} 7.4,100 \mathrm{mM} \mathrm{NaCl}, 2 \mathrm{mM} \mathrm{DTT}, 10 \mathrm{mM} \mathrm{ATP}$, and $10 \mathrm{mM} \mathrm{MgCl}_{2}$, and incubated at $37^{\circ} \mathrm{C}$. The $939500 \mu \mathrm{M}$ ubiquitin and $10 \mathrm{mM}$ ATP were added in small amounts every 30 min over the first $5 \mathrm{~h}$. 940 The reaction was then kept at $37^{\circ} \mathrm{C}$ overnight. The samples were incubated with Ultra HBC 941 streptavidin agarose beads (Goldbio) or FLAG antibody M2 agarose resin (Sigma) for $60 \mathrm{~min}$ at

$9424^{\circ} \mathrm{C}$. The resin was washed three times with 5 volumes of ubiquitination buffer and bound 943 material eluted with 5 volume of ubiquitination buffer containing $2 \mathrm{mM}$ biotin or $1 \mathrm{mg} / \mathrm{ml}$ 944 3xFLAG peptide. Subsequent gel filtration, SDS-PAGE analysis, and concentration determination 945 were performed as described above. To generate polyubiquitin chains containing Dendra$946 \mathrm{hUb}^{\mathrm{K} 48 \mathrm{R}}$, the ubiquitination reaction was first carried out with $1 \mu \mathrm{M}$ mouse Ube1, $20 \mu \mathrm{M}$ 947 gp78 ${ }^{\mathrm{RING}}$-Ube2g2, and $500 \mu \mathrm{M}$ wild-type human ubiquitin at $30^{\circ} \mathrm{C}$ for 5 h. $20 \mu \mathrm{M}$ Dendra$948 \mathrm{hUb}^{\mathrm{K} 48 \mathrm{R}}$ was then added to the reaction prior to the overnight incubation.

\section{HDX MS measurements}

951 Photoconverted, polyubiquitinated SBP-Dendra substrates were bound to streptavidin agarose 952 resin (Thermo) and incubated at $4^{\circ} \mathrm{C}$ with Ufd1, Npl4, and Cdc48 at a 1:1:1 molar ratio in 953 assembly buffer (50 mM HEPES, pH 7.5, $100 \mathrm{mM} \mathrm{NaCl}, 5 \mathrm{mM} \mathrm{MgCl}$, $1 \mathrm{mM} \mathrm{DTT}$ ). Note that no 954 nucleotides were present during the incubation. The resin was washed to remove unbound 955 Cdc48/UN complex. The beads were washed once with four bead volumes of assembly buffer 956 and then washed twice with four volumes of HDX equilibration buffer (20 mM Tris, $150 \mathrm{mM}$ $957 \mathrm{NaCl}, 5 \mathrm{mM} \mathrm{MgCl}_{2}, 0.5 \mathrm{mM} \mathrm{TCEP}, 100 \% \mathrm{H}_{2} \mathrm{O}, \mathrm{pH}$ 7.5). Bound protein was eluted with the HDX 958 equilibration buffer containing $2 \mathrm{mM}$ biotin, and concentrated. The final sample contained 
about $15 \mu \mathrm{M}$ Cdc48, $16 \mu \mathrm{M}$ UN, and $36 \mu \mathrm{M}$ Ub(n)-Dendra. As controls, the Cdc48/UN complex and photoconverted, polyubiquitinated SBP-Dendra were also buffer-exchanged separately into HDX equilibration buffer. $8 \mu \mathrm{M}$ of Cdc48/UN, Ub(n)-Dendra alone, or the Cdc48/UN in complex with Ub(n)-Dendra were mixed with $10 \mathrm{mM}$ of ADP or ATP and incubated at $30^{\circ} \mathrm{C}$ for $30 \mathrm{~min}$, and then kept on ice until HDX began.

Deuterium labeling and measurement were performed as previously described (Twomey et al., 2019). To initiate HDX, $1.0 \mu \mathrm{l}$ of each protein sample was diluted at $20^{\circ} \mathrm{C}$ with $18 \mu \mathrm{l}$ labeling buffer (20 mM Tris- $\mathrm{HCl}, 150 \mathrm{mM} \mathrm{NaCl}, 5 \mathrm{mM} \mathrm{MgCl}_{2}$, and $0.5 \mathrm{mM} \mathrm{TCEP}, 99.9 \% \mathrm{D}_{2} \mathrm{O}$, pD 7.4). At each labeling time ( $5 \mathrm{~s}, 10 \mathrm{~s}, 1 \mathrm{~min}, 10 \mathrm{~min}$, and $4 \mathrm{~h}$ ), $19 \mu \mathrm{l}$ of quench buffer was added (150 $\mathrm{mM}$ potassium phosphate $\mathrm{pH} 2.49, \mathrm{H}_{2} \mathrm{O}$ ). All subsequent steps were performed at $0{ }^{\circ} \mathrm{C}$. Quenched samples were digested online with immobilized pepsin using a Waters UPLC

971 instrument with HDX technology (Wales et al., 2008), desalted, and then eluted into a Waters

972 Synapt XS mass spectrometer with a 5-35\% gradient of water:acetonitrile over 10 min. Peptic

973 peptides were identified using ProteinLynx Global Server (PLGS) 3.0.1 (Waters) and deuterium

974 incorporation measured using DynamX 3.0 (Waters). The deuterium levels were not corrected

975 for back exchange and are reported as relative (Wales and Engen, 2006). The error of

976 measuring deuterium in this LC/MS setup was +/- 0.20 relative Da and differences were considered meaningful if they were larger than 0.50 Da. The recommended summary (Masson

978 et al., 2019) of HDX MS experimental parameters, proteolytic maps for all proteins, and the numeric values used to create HDX-MS figures are provided in Table S1. The raw HDX MS data and expanded technical details of the HDX MS acquisition and data processing steps have been deposited to the ProteomeXchange Consortium via the PRIDE partner repository (Perez-

982 Riverol et al., 2019) with the dataset identifier PXD027639.

\section{Ubiquitin modification by a maleimide-conjugated fluorescent dye}

985 A cysteine-free substrate containing the $\mathrm{N}$-end rule degron and photoconverted Dendra was 986 polyubiquitinated with purified $\mathrm{hUb}^{13 \mathrm{C}}$, as described above, resulting in $\mathrm{Ub}^{13 \mathrm{C}}(\mathrm{n})$-Dendra. $400 \mathrm{nM}$ 987 of $\mathrm{Ub}^{13 \mathrm{C}}(\mathrm{n})$-Dendra were incubated with $400 \mathrm{nM}$ Ufd1, $400 \mathrm{nM} \mathrm{Npl4}$, and $1 \mu \mathrm{M}$ full-length Cdc48 
or Cdc48 ${ }^{\mathrm{ND} 1}$ in SEC buffer containing $10 \mathrm{mM}$ ADP for $40 \mathrm{~min}$ at $4^{\circ} \mathrm{C}$. The samples were then supplemented with $100 \mu \mathrm{M}$ of maleimide-conjugated DyLight 680 dye (Thermo), incubated for another $10 \mathrm{~min}$, and quenched by the addition of $20 \mathrm{mM}$ DTT. The samples were mixed with SDS sample buffer and analyzed by SDS-PAGE, followed by fluorescence scanning on an Odyssey imager (LI-COR) and Coomassie-blue staining.

\section{Substrate unfolding assays}

995 With the exceptions mentioned below, substrate unfolding experiments were performed as 996 previously described (Twomey et al., 2019). Briefly, $400 \mathrm{nM}$ of the polyubiquitinated, 997 photoconverted Dendra or Eos proteins were mixed with $400 \mathrm{nM}$ UN variants and $400 \mathrm{nM}$ 998 Cdc48 variants in 50 mM HEPES pH 7.5, $150 \mathrm{mM} \mathrm{NaCl}, 10 \mathrm{mM} \mathrm{MgCl}$, $0.5 \mathrm{mM}$ TCEP, and 0.5 $999 \mathrm{mg} / \mathrm{ml}$ protease-free bovine serum albumin (BSA). After a 10 -min pre-incubation at $30^{\circ} \mathrm{C}$, an ATP regeneration mixture was added (10 mM ATP, $20 \mathrm{mM}$ phosphocreatine, $100 \mathrm{\mu g} / \mathrm{ml}$ creatine kinase), and the fluorescence (excitation, $540 \mathrm{~nm}$; emission, $580 \mathrm{~nm}$; gain, 80 to 100) was measured at 15-s intervals for 30 min, using a Synergy Neo2 Multi-mode reader (BioTek). Fluorescence of the unfolding reactions in Figures 4B, S2F, and S5A were measured at 30-S intervals for 30 min, using FlexStation 3 Microplate Reader (Molecular Devices).

The unfolding reactions in Figure $3 \mathrm{E}$ contained no TCEP. $800 \mathrm{nM}$ of the oxidized Cdc48 ${ }^{2 \mathrm{Cys}}$ was pre-treated with $20 \mathrm{mM}$ DTT to generate the reduced form of $\mathrm{Cdc} 48^{2 \mathrm{Cys}}$. For protease treatment of polyubiquitinated substrates, $2 \mu \mathrm{M}$ of substrates were incubated with $25 \mu \mathrm{M}$ TEV protease (Figure 3F) or $9 \mu \mathrm{M}$ Ulp1 (Figure S2F) in SEC buffer containing $1 \mathrm{mM}$ DTT at 30 ${ }^{\circ} \mathrm{C}$ for 1 $\mathrm{h}$, before being added to the unfolding reactions. Unfolding reactions in Figure $5 \mathrm{C}$ contained 800 nM Ufd1/Npl4 and 800 nM Cdc48, the ones in Figure S5F contained 800 nM substrate, 800 $\mathrm{nM}$ Ufd1/Npl4, and the indicated concentrations of Cdc48.

1014 Unfolding assays performed in conjunction with pull-downs (Figures 6F, S5A-E) were described 1015 in the section "in vitro pull-down experiments". Unfolding reactions in Figure S5C-D were 1016 scanned in dual fluorescence mode with an additional green fluorescence channel (excitation 2, 
$1017488 \mathrm{~nm}$; emission 2, $525 \mathrm{~nm}$; gain 2, 80). Unfolding assays in conjunction with photocrosslinking

1018 (Figures 4B and S7A) were described in the section "photocrosslinking experiments". Unfolding 1019 reactions in Figure S7E were carried out with 400 nM Ub ${ }^{13 C}(n)$-Dendra, 400 nM HA-Ufd1 ${ }^{\text {TEV }}, 400$ $1020 \mathrm{nM}$ Npl4-FLAG, $1 \mu \mathrm{M}$ Cdc48, and $10 \mathrm{mM}$ of the indicated nucleotides. Protease-free BSA was 1021 omitted to avoid competition of BSA with the maleimide-conjugated DyLight 680 dye. After 1022 unfolding, the samples were incubated on ice for $1 \mathrm{~h}$ in the presence or absence of $1 \mu \mathrm{M}$ TEV 1023 protease, prior to the addition of the maleimide-conjugated dye. Subsequent ubiquitin 1024 modification assays were performed as described above.

1025

1026 For data acquired on Synergy Neo2 Multi-mode reader (BioTek), the relative fluorescence at 1027 time $\mathrm{t}$ was calculated as (fluorescence at $\mathrm{t}$ ) / (fluorescence at $\mathrm{t}_{0}$ ). For each experiment 1028 performed on FlexStation 3 Microplate Reader (Molecular Devices), an empty well was included 1029 to determine background fluorescence. The relative fluorescence at time t was calculated as 1030 [(fluorescence at $\mathrm{t}$ ) - (background fluorescence at $\mathrm{t})]$ / [(fluorescence at $\left.\mathrm{t}_{0}\right)$ - (background 1031 fluorescence at $\left.t_{0}\right)$ ]. The calculated relative fluorescence was plotted against time using Prism 1032 software (GraphPad). A linear fit was performed with data points within the first 2 min to 1033 calculate the initial velocities. These rates were normalized to that of the wild-type sample in 1034 the same experiment. Inhibition of substrate unfolding was determined by the reduction of the 1035 initial unfolding velocity compared to the wild-type sample. See Table $\mathbf{S 2}$ for the raw data of all 1036 substrate unfolding assays.

\section{In vitro pull-down experiments}

1039 For all in-vitro pull-down experiments, with the exceptions mentioned below, $1 \mu \mathrm{M}$ 1040 polyubiquitinated substrate was mixed with $1 \mu \mathrm{M}$ Cdc48, $1 \mu \mathrm{M}$ Ufd1, and/or $1 \mu \mathrm{M}$ Npl4 in 1041 binding buffer (50 mM Tris- $\mathrm{HCl}, \mathrm{pH}$ 8, $150 \mathrm{mM} \mathrm{NaCl}, 10 \mathrm{mM} \mathrm{MgCl}_{2}$, and $1 \mathrm{mM} \mathrm{DTT}$ )

1042 supplemented with $10 \mathrm{mM}$ of the desired nucleotide. $50 \mu \mathrm{l}$ of such a protein mixture were then 1043 incubated with $8 \mu$ l pre-equilibrated FLAG antibody M2 agarose beads (Sigma) or streptavidin 1044 agarose beads (Thermo) at $4^{\circ} \mathrm{C}$ for $1 \mathrm{~h}$. The beads were then washed three times with binding 1045 buffer containing the desired nucleotide. Bead-bound proteins were eluted with $25 \mu$ of 
binding buffer supplemented with $0.05 \%$ Tween-20 and either $0.2 \mathrm{mg} / \mathrm{ml} 3 x \mathrm{FLAG}$ peptide

1047 (Bimake) or $2 \mathrm{mM}$ biotin (Sigma). The eluted samples were subjected to SDS-PAGE, followed by

1048 fluorescence scanning on an Odyssey imager (LI-COR) and Coomassie-blue staining. When

1049 oxidized $\mathrm{Cdc} 48^{2 \mathrm{Cys}}$ was used for pull-downs, all proteins were buffer-exchanged into non-

1050 reducing SEC buffer, and the binding reactions were carried out in a non-reducing binding

1051 buffer (50 mM Tris- $\mathrm{HCl}, \mathrm{pH} 8,150 \mathrm{mM} \mathrm{NaCl}$, and $10 \mathrm{mM} \mathrm{MgCl}$ ).

1052

1053 For the substrate-dissociation assay shown in Figures 6C-D, substrate unfolding reactions were 1054 performed with $200 \mathrm{nM}$ substrate, $1 \mu \mathrm{M}$ Cdc48, $1 \mu \mathrm{M}$ Ufd1 ${ }^{\text {TEV }}, 1 \mu \mathrm{M}$ Npl4-FLAG, and $10 \mathrm{mM}$ of 1055 ADP or ATP. $50 \mu$ l of each reaction were then incubated with $5 \mu$ l pre-equilibrated FLAG 1056 antibody $\mathrm{M} 2$ agarose beads (Sigma) at $4^{\circ} \mathrm{C}$ for $1 \mathrm{~h}$. After washing with nucleotide-free binding 1057 buffer, the beads were resuspended in $50 \mu$ l unfolding buffer containing $4.5 \mu \mathrm{M}$ TEV protease 1058 and $10 \mathrm{mM}$ of the desired nucleotide, and the incubation was continued at room temperature 1059 for another $2 \mathrm{~h}$. After three washes with the binding buffer supplemented with appropriate 1060 nucleotides, the bound proteins were eluted and analyzed as described above.

1062 For the substrate exchange experiment shown in Figure 6E, the unfolding reactions were 1063 performed with $1 \mu \mathrm{M}$ DyLight 800-labeled substrate, $2 \mu \mathrm{M}$ Cdc48-FLAG, $4 \mu \mathrm{M}$ SBP-Ufd1, $4 \mu \mathrm{M}$ $1064 \mathrm{Npl}$, and $10 \mathrm{mM}$ of ADP or ATP. $50 \mu \mathrm{l}$ of each reaction were then incubated with $5 \mu$ pre1065 equilibrated streptavidin agarose beads (Thermo) at $4^{\circ} \mathrm{C}$ for $1 \mathrm{~h}$. After three washes with 1066 assembly buffer (50 mM HEPES, pH 7.5, $100 \mathrm{mM} \mathrm{NaCl}, 5 \mathrm{mM} \mathrm{MgCl}$, 1 mM DTT), bound

1067 proteins were eluted by $2 \mathrm{mM}$ biotin in assembly buffer. The eluted proteins were mixed with 1 $1068 \mu \mathrm{M}$ DyLight 680-labeled substrate and $10 \mathrm{mM}$ of the desired nucleotide for a second unfolding 1069 reaction. The samples were then incubated with $5 \mu \mathrm{l}$ pre-equilibrated FLAG antibody M2 1070 agarose beads (Sigma) for $1 \mathrm{~h}$ at $4^{\circ} \mathrm{C}$, followed by washing, elution, and SDS-PAGE, as described 1071 above.

1072

1073 Photocrosslinking experiments 
1074 The photocrosslinking experiments in Figures S2D, S3D, and S3I were performed as described 1075 (Twomey et al., 2019), with some modifications. Briefly, the reaction components included

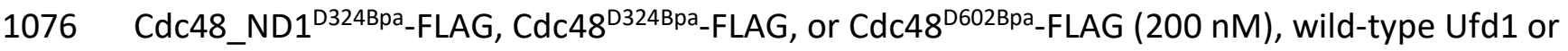
1077 Ufd1 $\Delta$ UT3 (500 nM), wild-type or mutant Npl4 (500 nM), and dye-labeled polyubiquitinated 1078 sfGFP $(1 \mu \mathrm{M})$ in reaction buffer $(50 \mathrm{mM}$ HEPES pH 7.5, $150 \mathrm{mM} \mathrm{NaCl}, 10 \mathrm{mM} \mathrm{MgCl}$, $0.5 \mathrm{mM}$ 1079 TCEP, $0.5 \mathrm{mg} / \mathrm{ml}$ protease-free BSA) supplemented with $10 \mathrm{mM}$ of ADP. The reactions were 1080 assembled on ice, incubated at $30^{\circ} \mathrm{C}$ for $10 \mathrm{~min}$, and transferred to individual PCR tubes. A long1081 wave UV lamp (Blak-Ray) was positioned $5 \mathrm{~cm}$ above the tubes, and the samples were 1082 irradiated for $30 \mathrm{~min}$. To prevent overheating, an ice-cold metal block was placed in contact 1083 with the bottom of the PCR tubes. After irradiation, the samples were diluted 10 -fold in 1084 dissociation buffer (50 mM Tris- $\mathrm{HCl}, \mathrm{pH} 8,800 \mathrm{mM} \mathrm{NaCl}, 1 \%(\mathrm{v} / \mathrm{v})$ Triton X-100, 1 mM EDTA, 0.5 $1085 \mathrm{mM} \mathrm{DTT}$ ) and incubated at room temperature for $5 \mathrm{~min}$. The samples were then applied to $8 \mu \mathrm{l}$ 1086 FLAG antibody M2 magnetic beads (Sigma) equilibrated in dissociation buffer for $1 \mathrm{~h}$ at room 1087 temperature. The beads were washed three times, and bound protein was eluted in $50 \mathrm{mM}$ 1088 HEPES pH 7.5, $150 \mathrm{mM} \mathrm{NaCl}, 0.5 \mathrm{mM}$ TCEP, and $0.2 \mathrm{mg} / \mathrm{ml}$ 3xFlag peptide (Bimake). The eluted 1089 material was subjected to SDS-PAGE and the gel scanned on an Odyssey imager (LI-COR). The gel was then transferred to a nitrocellulose membrane and analyzed by immunoblotting with Cdc48 antibodies on an Amersham Imager 600 RGB (Cytiva). SBP-Ufd1, 1.25 $\mu \mathrm{M}$ FLAG-tagged Npl4 Bpa mutant, and 1.25 $\mu \mathrm{M}$ dye-labeled, photoconverted, and polyubiquitinated Dendra, in $50 \mathrm{mM}$ HEPES, pH 7.5, $100 \mathrm{mM} \mathrm{NaCl}, 5 \mathrm{mM} \mathrm{MgCl}$, and $1 \mathrm{mM}$ DTT. $16 \mu$ l of the protein mixture were then diluted with $29 \mu$ l of unfolding buffer $(50 \mathrm{mM}$ 1097 HEPES, pH 7.5, $150 \mathrm{mM} \mathrm{NaCl}, 10 \mathrm{mM} \mathrm{MgCl}, 0.5 \mathrm{mg} / \mathrm{ml}$ protease-free BSA). After a 10-min pre1098 incubation at $30^{\circ} \mathrm{C}, 5 \mu$ of ATP $(100 \mathrm{mM})$ was added and the fluorescence (excitation, $540 \mathrm{~nm}$; 1099 emission, $580 \mathrm{~nm}$ ) was measured at 30-s intervals for $30 \mathrm{~min}$, using a FlexStation 3 Microplate 1100 Reader (Molecular Devices). Both crosslinked and non-crosslinked unfolding reactions were 1101 analyzed by SDS-PAGE. The non-crosslinked sample was also subjected to UV irradiation after 1102 substrate unfolding, and analyzed by SDS-PAGE side-by-side, as shown in Figure S4B. 
1104 For photocrosslinking experiment followed by MS (Figures S7A-D), an unfolding assay was first 1105 performed with 400 nM Cdc48 ${ }^{\text {D602Bpa }}$-FLAG, 600 nM Ufd1/Npl4, 400 nM dye-labeled, photoconverted, and polyubiquitinated Dendra, and 10 mM ADP or ATP. Unfolding of Dendra was

1107 monitored at 15-s intervals for 30 min using a Synergy Neo2 Multi-mode reader (BioTek). The 1108 samples were then transferred to a PCR tube for UV irradiation. The Cdc48 ${ }^{\mathrm{D} 602 \mathrm{Bpa}}$-FLAG protein 1109 was isolated by FLAG antibody M2 magnetic beads (Sigma) and bound material eluted with 0.2 $1110 \mathrm{mg} / \mathrm{ml}$ single FLAG peptide (Sigma-Aldrich) in $50 \mathrm{mM}$ Tris- $\mathrm{HCl}, \mathrm{pH} \mathrm{8,} 150 \mathrm{mM} \mathrm{NaCl}, 0.05 \%$ NP1111 40, 1 mM EDTA, 10\% glycerol, and protease inhibitor cocktail. The eluted material was 1112 subjected to both SDS-PAGE and MS analysis.

\section{MS of photocrosslinked proteins}

1115 The analysis of crosslinked peptides was performed by nano-liquid chromatography and 1116 tandem MS, as previously described (Twomey et al., 2019), with minor modifications. FLAG 1117 peptide eluates were diluted 1:1 with $100 \mathrm{mM}$ ammonium bicarbonate, denatured with $0.1 \%$ 1118 Rapigest (Waters Corporation, Milford, MA), reduced with $10 \mathrm{mM} \mathrm{DTT}$ for 30 min at $56^{\circ} \mathrm{C}$, 1119 cooled for $5 \mathrm{~min}$ at room temperature, alkylated with $22.5 \mathrm{mM}$ iodoacetamide for $30 \mathrm{~min}$ at 1120 room temperature protected from light, and then digested with trypsin overnight at $37^{\circ} \mathrm{C}$.

1121 Rapigest was cleaved by adding trifluoroacetic acid to a final concentration of $1 \%$ and 1122 incubating for an additional $30 \mathrm{~min}$ at $37^{\circ} \mathrm{C}$. After centrifugation to remove Rapigest by1123 products, peptides in the supernatant were desalted using C18 (SOLA-RP, ThermoFisher

1124 Scientific, Madison, WI). C18 eluates were dried by vacuum centrifugation, and residual

1125 detergent was removed using magnetic beads (Hughes et al., 2014).

1127 Peptides were analyzed by nanoLC-MS as described (Ficarro et al., 2009) using a NanoAcquity 1128 UPLC system (Waters Corporation) interfaced to a QExactive HF mass spectrometer 1129 (ThermoFisher Scientific). Peptides were injected onto a self-packed pre-column (100 $\mu \mathrm{m}$ I.D. 1130 packed with $4 \mathrm{~cm}$ POROS 10R2, Applied Biosystems, Framingham, MA), resolved on an analytical 1131 column (30 $\mathrm{mm}$ I.D. packed with $50 \mathrm{~cm} 5 \mu \mathrm{m}$ Monitor C18, Orochem, Naperville, IL), and 
1132 introduced to the mass spectrometer via ESI (spray voltage $=4 \mathrm{kV}$ ). The mass spectrometer was

1133 programmed to operate in data dependent mode, such that the 15 most abundant precursor

1134 ions in each MS scan ( $\mathrm{m} / \mathrm{z}$ 300-2000, 120K resolution, target=1E6) were subjected to MS/MS

1135 (target value=5E4, max fill time $=50 \mathrm{~ms}$, isolation width=1.6 $\mathrm{Da}$, resolution=15K, collision 1136 energy=30\%).

1138 Cross-linked peptides were identified using CrossFinder version 1.4 (Mueller-Planitz, 2015) to 1139 search against a customized protein sequence database consisting of Cdc48, Ufd1, Npl4, 1140 ubiquitin, and the fusion protein of $\mathrm{N}$-end-rule degron and the fluorescent protein Dendra.

\section{Immunoprecipitation of yeast lysates}

1143 Immunoprecipitation (IP) from lysates of S. cerevisiae cells was performed as described in 1144 (Tsuchiya et al., 2017) with some modifications. Briefly, a cell pellet corresponding to 120 $1145 \mathrm{OD}_{600}$ was resuspended in $800 \mu$ l of lysis buffer $(50 \mathrm{mM}$ Tris- $\mathrm{HCl}, \mathrm{pH} 7.5,100 \mathrm{mM} \mathrm{NaCl}, 10 \%$ 1146 glycerol, $10 \mu \mathrm{M}$ bortezomib, $10 \mathrm{mM}$ iodoacetomide, 1x Protease Inhibitor Cocktail (Roche)) and 1147 mixed with $1 \mathrm{ml}$ of acid-washed glass beads. The cells were lysed using a Mini-BeadBeater 96 1148 (BIOSPEC) at 2,400 rpm for $4.5 \mathrm{~min}$. The cell lysate was then spun at $500 \mathrm{~g}$ for $3 \mathrm{~min}$ at $4^{\circ} \mathrm{C}$ to 1149 remove unbroken cells and debris. The supernatant was supplemented with 1\% TX-100 and 1150 incubated on ice for $30 \mathrm{~min}$, before spinning at 20,000 $\mathrm{g}$ for $20 \mathrm{~min}$. A small aliquot of the 1151 cleared lysate was used for immunoblots with K48-specific ubiquitin antibodies and anti-PGK1 1152 antibodies. The protein concentration in the cleared lysate was determined with a Protein 1153 Assay Dye Reagent (BioRad). One half of the lysate containing $2.5 \mathrm{mg}$ of proteins was 1154 incubated with $25 \mu$ l FLAG antibody agarose resin (Sigma, M2) that was pre-equilibrated with 1155 lysis buffer containing 1\% TX-100. The other half of the lysate was incubated with $5 \mu \mathrm{g}$ of 1156 biotinylated, K48-specific TUBE (LifeSensors, UM307) and $25 \mu$ pre-equilibrated streptavidin 1157 agarose beads (ThermoFisher). After one hour incubation at $4^{\circ} \mathrm{C}$, the beads were washed three 1158 times with $1 \mathrm{ml}$ of the lysis buffer supplemented with $1 \% \mathrm{TX}-100$. Bound material was eluted 1159 with 4 bead-volumes of elution buffer (50 mM HEPES, pH 7.4, $150 \mathrm{mM} \mathrm{NaCl}, 5 \mathrm{mM} \mathrm{MgCl}$, 0.5 $1160 \mathrm{mM}$ TCEP, $0.1 \%$ TX-100) supplemented with $1 \mathrm{mg} / \mathrm{ml}$ TR-TUBE. $20 \mu \mathrm{l}$ of the total eluate was 
1161 examined by SDS-PAGE, silver stain, and immunoblotting using K48-specific ubiquitin

1162 antibodies. The other $80 \mu$ of the eluate were precipitated by adding $20 \mu \mathrm{l}$ of $100 \%$

1163 trichloroacetic acid (TCA) (Sigma). After 30-min incubation on ice, the samples were centrifuged

1164 at $14,000 \mathrm{rpm}$ for $15 \mathrm{~min}$ at $4^{\circ} \mathrm{C}$. The pellet was sequentially washed with $1 \mathrm{ml}$ of ice-cold

1165 acetone and $1 \mathrm{ml}$ of cold methanol. The protein pellet was then resuspended and digested with

1166 trypsin for MS analysis.

\section{TMT labeling}

1169 TMTpro reagents $(0.8 \mathrm{mg})$ were dissolved in anhydrous acetonitrile $(40 \mu \mathrm{l})$ of which $7 \mu \mathrm{l}$ were 1170 added to the peptides $(50 \mu \mathrm{g})$. Acetonitrile $(13 \mu \mathrm{l})$ was added to achieve a final concentration

1171 of approximately $30 \%(\mathrm{v} / \mathrm{v})$. Following incubation at room temperature for $1 \mathrm{~h}$, the reaction was

1172 quenched with hydroxylamine at a final concentration of $0.3 \%(v / v)$. Equal amounts of all

1173 TMTpro-labeled samples were pooled, dried under vacuum in a SpeedVac, and subjected to a

1174 C18 solid-phase extraction (SPE) column with a capacity of $100 \mathrm{mg}$ (Sep-Pak, Waters).

1176 Liquid chromatography and tandem MS

1177 MS data were collected on an Orbitrap Eclipse mass spectrometer coupled to a Proxeon

1178 NanoLC-1200 UHPLC. The $100 \mu \mathrm{m}$ capillary column was packed with $35 \mathrm{~cm}$ of Accucore 150

1179 resin $(2.6 \mu \mathrm{m}, 150 \AA$; ThermoFisher Scientific). Data were acquired for $180 \mathrm{~min}$ for a total of 4

1180 injections of the unfractionated sample. The scan sequence began with an MS1 spectrum

1181 (Orbitrap analysis, resolution 120,000, 400-1500 Th, automatic gain control (AGC) target was

1182 set to "standard", maximum injection time was set to "auto"). The number of data dependent

1183 scans was set to 20. MS2 analysis consisted of higher-energy collision-activated dissociation

1184 (HCD), the Orbitrap resolution was set at 50,000, the isolation window was $0.7 \mathrm{Da}$, automatic 1185 gain control (AGC) was set at $300 \%$, and HCD collision energy was $37 \%$. All data were acquired

1186 with FAIMS with the dispersion voltage (DV) set at 5000V. Injections differed in the

1187 compensation voltages (CVs) used. One sample was subjected to $C V=-40,-60$, and $-80 \mathrm{~V}$, a

1188 second sample with -50 and $-70 \mathrm{~V}$ and two samples with $-45,-55,-65$, and $-75 \mathrm{~V}$. In one of the

1189 samples with the four CVs, the precursor priority was set from least intense to most intense. 
Spectra were converted to mzXML via MSconvert (Chambers et al., 2012). Database searching included all entries from the yeast UniProt Database (downloaded: August 2020). The database was concatenated with one composed of all protein sequences for that database in

1194 the reversed order. Searches were performed using a 50-ppm precursor ion tolerance for total protein level profiling. The product ion tolerance was set to $0.02 \mathrm{Da}$. These wide mass tolerance windows were chosen to maximize sensitivity in conjunction with Comet searches and linear

1197 discriminant analysis (Beausoleil et al., 2006; Huttlin et al., 2010). TMTpro labels on lysine 1198 residues and peptide $\mathrm{N}$-termini (+304.207 Da), as well as carbamidomethylation of cysteine 1199 residues (+57.021 Da) were set as static modifications, while oxidation of methionine residues 1200 (+15.995 Da) was set as a variable modification. Peptide-spectrum matches (PSMs) were 1201 adjusted to a 1\% false discovery rate (FDR) (Elias and Gygi, 2007; 2010). PSM filtering was performed using a linear discriminant analysis, as described previously (Huttlin et al., 2010) and then assembled further to a final protein-level FDR of 1\% (Elias and Gygi, 2007). Proteins were quantified by summing reporter ion counts across all matching PSMs, also as described

1205 previously (McAlister et al., 2012). Reporter ion intensities were adjusted to correct for the 1206 isotopic impurities of the different TMTpro reagents according to manufacturer specifications.

1207 The signal-to-noise (S/N) measurements of peptides assigned to each protein were summed and these values were normalized so that the sum of the signal for all proteins in each channel

1209 was equivalent to account for equal protein loading. Finally, each protein abundance measurement was scaled, such that the summed signal-to-noise for that protein across all

1211 channels equals 100, thereby generating a relative abundance measurement.

1213 For each substrate protein detected, the ratio of its abundance in the FLAG IP versus K48 IP 1214 from the same cell lysate was calculated. The two ratios calculated from cell lysates under the 1215 same condition were averaged as the $\mathrm{Cdc48/K48} \mathrm{ratio.} \mathrm{The} \mathrm{averaged} \mathrm{Cdc48/K48} \mathrm{ratios} \mathrm{of} \mathrm{all}$ 1216 substrate proteins from four different conditions - Npl4-FLAG/DMSO, Ufd1-FLAG/DMSO, Npl41217 FLAG/bortezomib, and Ufd1-FLAG/bortezomib - were then plotted as pairwise comparisons in 1218 a logarithmic scale. After confirming the overall distributions of Cdc48/K48 ratio across all the 
1219 four conditions were very similar, the Cdc48/K48 ratios of each substrate protein were further

1220 averaged. The resulting Cdc48/K48 ratios were plotted in a logarithmic scale to generate a

1221 distribution plot. All graphs were generated using Prism software (GraphPad).

1222

1223

1224

1225

\section{QUANTIFICATION AND STATISTICAL ANALYSIS}

1226 Quantifications of fluorescence scanning gels were carried out using the ImageStudio software

1227 (LI-COR). For each lane, a rectangle box was selected to determine total intensity of a band or

1228 smear of signal. The rectangle boxes for all lanes on the same gel were kept with similar box

1229 sizes. For each gel, an additional rectangle box with similar box size were drawn over an empty

1230 or non-signal region to determine background intensity. Signal intensity of each lane was

1231 calculated as (total intensity - box size * background intensity / background box size). The

1232 resulting signal intensity was normalized to a designated lane to calculate the relative signal

1233 intensity.

1234

1235 Band intensities on Coomassie blue-stained gels were quantified using ImageJ (NIH).

1236 Background subtraction and normalization were performed the same as fluorescence scanning 1237 gels described above. 


\section{SUPPLEMENTAL INFORMATION}

1243

1244 Supplemental information includes:

1245 Document S1. Figures S1-S7

1246 Table S1. Hydrogen-deuterium exchange mass spectrometry data (related to Figures 2 and S6)

1247 Table S2. Raw data of substrate unfolding assays (related to Figures 3-6, S2, S3, S5, and S7)

1248

1249

1250

1251 


\section{REFERENCES}

1253 Anderson, D.J., Le Moigne, R., Djakovic, S., Kumar, B., Rice, J., Wong, S., Wang, J., Yao, B., Valle, 1254 E., Kiss von Soly, S., et al. (2015). Targeting the AAA ATPase p97 as an Approach to Treat Cancer 1255 through Disruption of Protein Homeostasis. Cancer Cell 28, 653-665.

1256 10.1016/j.ccell.2015.10.002.

1257 Avellaneda, M.J., Franke, K.B., Sunderlikova, V., Bukau, B., Mogk, A., and Tans, S.J. (2020). 1258 Processive extrusion of polypeptide loops by a Hsp100 disaggregase. Nature 578, 317-320. 1259 10.1038/s41586-020-1964-y.

Beausoleil, S.A., Villén, J., Gerber, S.A., Rush, J., and Gygi, S.P. (2006). A probability-based approach for high-throughput protein phosphorylation analysis and site localization. Nat. 1262 Biotechnol. 24, 1285-1292. 10.1038/nbt1240.

Blythe, E.E., Olson, K.C., Chau, V., and Deshaies, R.J. (2017). Ubiquitin- and ATP-dependent unfoldase activity of P97/VCP•NPLOC4 $\bullet U F D 1 L$ is enhanced by a mutation that causes multisystem proteinopathy. Proc. Natl. Acad. Sci. USA. 114, E4380-E4388. 10.1073/pnas.1706205114.

Bodnar, N., and Rapoport, T. (2017a). Toward an understanding of the Cdc48/p97 ATPase. F1000Res. 6, 1318. 10.12688/f1000research.11683.1.

Bodnar, N.O., and Rapoport, T.A. (2017b). Molecular mechanism of substrate processing by the Cdc48 ATPase complex. Cell 169, 722-735.e9. 10.1016/j.cell.2017.04.020.

1271 Chambers, M.C., Maclean, B., Burke, R., Amodei, D., Ruderman, D.L., Neumann, S., Gatto, L., 1272 Fischer, B., Pratt, B., Egertson, J., et al. (2012). A cross-platform toolkit for mass spectrometry 1273 and proteomics. Nat. Biotechnol. 30, 918-920. 10.1038/nbt.2377.

1274 Chau, V., Tobias, J.W., Bachmair, A., Marriott, D., Ecker, D.J., Gonda, D.K., and Varshavsky, A. 1275 (1989). A multiubiquitin chain is confined to specific lysine in a targeted short-lived protein.

1277 Chin, J.W., Martin, A.B., King, D.S., Wang, L., and Schultz, P.G. (2002). Addition of a photocrosslinking amino acid to the genetic code of Escherichiacoli. Proc. Natl. Acad. Sci. USA. $127999,11020-11024.10 .1073 /$ pnas.172226299.

1280 Elias, J.E., and Gygi, S.P. (2007). Target-decoy search strategy for increased confidence in largescale protein identifications by mass spectrometry. Nat. Methods 4, 207-214.

$128210.1038 /$ nmeth1019.

1283 Elias, J.E., and Gygi, S.P. (2010). Target-decoy search strategy for mass spectrometry-based 1284 proteomics. Methods Mol. Biol. 604, 55-71. 10.1007/978-1-60761-444-9_5. 
Ficarro, S.B., Zhang, Y., Lu, Y., Moghimi, A.R., Askenazi, M., Hyatt, E., Smith, E.D., Boyer, L., Schlaeger, T.M., Luckey, C.J., et al. (2009). Improved electrospray ionization efficiency compensates for diminished chromatographic resolution and enables proteomics analysis of tyrosine signaling in embryonic stem cells. Anal. Chem. 81, 3440-3447. 10.1021/ac802720e.

1289 Finley, D., Ulrich, H.D., Sommer, T., and Kaiser, P. (2012). The ubiquitin-proteasome system of 1290 Saccharomyces cerevisiae. Genetics 192, 319-360. 10.1534/genetics.112.140467.

1291 Fleming, J.A., Lightcap, E.S., Sadis, S., Thoroddsen, V., Bulawa, C.E., and Blackman, R.K. (2002). 1292 Complementary whole-genome technologies reveal the cellular response to proteasome inhibition by PS-341. Proc. Natl. Acad. Sci. USA. 99, 1461-1466. 10.1073/pnas.032516399.

1294 Greene, E.R., Dong, K.C., and Martin, A. (2020). Understanding the 26S proteasome molecular 1295 machine from a structural and conformational dynamics perspective. Curr. Opin. Struct. Biol. 1296 61, 33-41. 10.1016/j.sbi.2019.10.004.

1297 Han, H., Fulcher, J.M., Dandey, V.P., Iwasa, J.H., Sundquist, W.I., Kay, M.S., Shen, P.S., and Hill, 1298 C.P. (2019). Structure of Vps4 with circular peptides and implications for translocation of two 1299 polypeptide chains by AAA+ATPases. eLife 8, e44071. 10.7554/eLife.44071.

Hänzelmann, P., and Schindelin, H. (2017). The Interplay of Cofactor Interactions and Posttranslational Modifications in the Regulation of the AAA+ ATPase p97. Front. Mol. Biosci. 4, 21. 10.3389/fmolb.2017.00021. Ultrasensitive proteome analysis using paramagnetic bead technology. Mol. Syst. Biol. 10, 757. $10.15252 / \mathrm{msb} .20145625$. W., Sowa, M.E., and Gygi, S.P. (2010). A tissue-specific atlas of mouse protein phosphorylation and expression. Cell 143, 1174-1189. 10.1016/j.cell.2010.12.001. ubiquitin. Proc. Natl. Acad. Sci. USA. 102, 13427-13432. 10.1073/pnas.0501581102.

Johnson, J.O., Mandrioli, J., Benatar, M., Abramzon, Y., Van Deerlin, V.M., Trojanowski, J.Q., Gibbs, J.R., Brunetti, M., Gronka, S., Wuu, J., et al. (2010). Exome sequencing reveals VCP mutations as a cause of familial ALS. Neuron 68, 857-864. 10.1016/j.neuron.2010.11.036. photoswitchable protein for oxidizing environments. Chem. Commun. (Camb) 53, 2106-2109. 10.1039/c6cc09997a.

1317 Kimonis, V.E., Fulchiero, E., Vesa, J., and Watts, G. (2008). VCP disease associated with 1318 myopathy, Paget disease of bone and frontotemporal dementia: review of a unique disorder. Biochimi. Biophys. Acta. 1782, 744-748. 10.1016/j.bbadis.2008.09.003. 
1320 Le Moigne, R., Aftab, B.T., Djakovic, S., Dhimolea, E., Valle, E., Murnane, M., King, E.M., Soriano, F., Menon, M.-K., Wu, Z.Y., et al. (2017). The p97 Inhibitor CB-5083 Is a Unique Disrupter of Protein Homeostasis in Models of Multiple Myeloma. Mol. Cancer Ther. 16, 2375-2386. 10.1158/1535-7163.MCT-17-0233.

Masson, G.R., Burke, J.E., Ahn, N.G., Anand, G.S., Borchers, C., Brier, S., Bou-Assaf, G.M., Engen, J.R., Englander, S.W., Faber, J., et al. (2019). Recommendations for performing, interpreting and reporting hydrogen deuterium exchange mass spectrometry (HDX-MS) experiments. Nat. Methods 16, 595-602. 10.1038/s41592-019-0459-y.

McAlister, G.C., Huttlin, E.L., Haas, W., Ting, L., Jedrychowski, M.P., Rogers, J.C., Kuhn, K., Pike, I., Grothe, R.A., Blethrow, J.D., et al. (2012). Increasing the multiplexing capacity of TMTs using reporter ion isotopologues with isobaric masses. Anal. Chem. 84, 7469-7478.

1332 Mueller-Planitz, F. (2015). Crossfinder-assisted mapping of protein crosslinks formed by site1333 specifically incorporated crosslinkers. Bioinformatics 31, 2043-2045.

1334 10.1093/bioinformatics/btv083.

1335

Park, S., Isaacson, R., Kim, H.T., Silver, P.A., and Wagner, G. (2005). Ufd1 exhibits the AAAATPase fold with two distinct ubiquitin interaction sites. Structure 13, 995-1005. 10.1016/j.str.2005.04.013.

Perez-Riverol, Y., Csordas, A., Bai, J., Bernal-Llinares, M., Hewapathirana, S., Kundu, D.J., Inuganti, A., Griss, J., Mayer, G., Eisenacher, M., et al. (2019). The PRIDE database and related tools and resources in 2019: improving support for quantification data. Nucleic Acids Res. 47, D442-D450. 10.1093/nar/gky1106.

Sato, Y., Tsuchiya, H., Yamagata, A., Okatsu, K., Tanaka, K., Saeki, Y., and Fukai, S. (2019). Structural insights into ubiquitin recognition and Ufd1 interaction of Npl4. Nat. Commun. 10, 5708-5713. 10.1038/s41467-019-13697-y.

Stein, A., Ruggiano, A., Carvalho, P., and Rapoport, T.A. (2014). Key steps in ERAD of luminal ER proteins reconstituted with purified components. Cell 158, 1375-1388.

10.1016/j.cell.2014.07.050.

Thrower, J.S., Hoffman, L., Rechsteiner, M., and Pickart, C.M. (2000). Recognition of the polyubiquitin proteolytic signal. EMBO J. 19, 94-102. 10.1093/emboj/19.1.94.

Tsuchiya, H., Ohtake, F., Arai, N., Kaiho, A., Yasuda, S., Tanaka, K., and Saeki, Y. (2017). In Vivo Ubiquitin Linkage-type Analysis Reveals that the Cdc48-Rad23/Dsk2 Axis Contributes to K48Linked Chain Specificity of the Proteasome. Mol. Cell 66, 488-502.e7. 10.1016/j.molcel.2017.04.024. 
1354 Twomey, E.C., Ji, Z., Wales, T.E., Bodnar, N.O., Ficarro, S.B., Marto, J.A., Engen, J.R., and 1355 Rapoport, T.A. (2019). Substrate processing by the Cdc48 ATPase complex is initiated by 1356 ubiquitin unfolding. Science 365, eaax1033. 10.1126/science.aax1033.

1357 van den Boom, J., and Meyer, H. (2018). VCP/p97-Mediated Unfolding as a Principle in Protein 1358 Homeostasis and Signaling. Mol. Cell 69, 182-194. 10.1016/j.molcel.2017.10.028.

1359 Wales, T.E., and Engen, J.R. (2006). Hydrogen exchange mass spectrometry for the analysis of 1360 protein dynamics. Mass Spectrom Rev. 25, 158-170. 10.1002/mas.20064.

1361 Wales, T.E., Fadgen, K.E., Gerhardt, G.C., and Engen, J.R. (2008). High-speed and high-resolution 1362 UPLC separation at zero degrees Celsius. Anal. Chem. 80, 6815-6820. 10.1021/ac8008862.

1363 Watts, G.D.J., Wymer, J., Kovach, M.J., Mehta, S.G., Mumm, S., Darvish, D., Pestronk, A., Whyte, 1364 M.P., and Kimonis, V.E. (2004). Inclusion body myopathy associated with Paget disease of bone 1365 and frontotemporal dementia is caused by mutant valosin-containing protein. Nat. Genet. 36, 1366 377-381. 10.1038/ng1332.

1367 Wu, X., and Rapoport, T.A. (2018). Mechanistic insights into ER-associated protein degradation. 1368 Curr. Opin. Cell Biol. 53, 22-28. 10.1016/j.ceb.2018.04.004.

1369 Yip, M.C.J., Bodnar, N.O., and Rapoport, T.A. (2020). Ddi1 is a ubiquitin-dependent protease. 1370 Proc. Natl. Acad. Sci. USA. 117, 7776-7781. 10.1073/pnas.1902298117. 
A $\quad$ BY4741: $p d r 5 \Delta$

Npl4-FLAG or Ufd1-FLAG
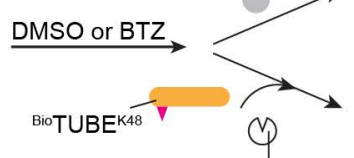

Streptavidin resin

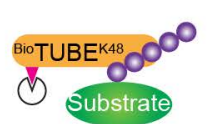

C
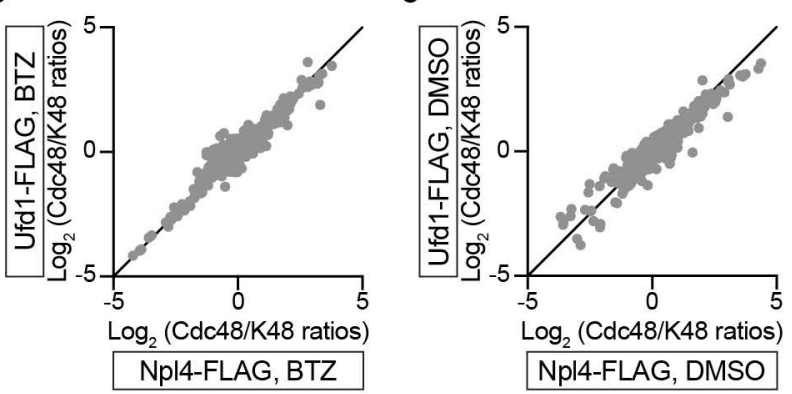

D

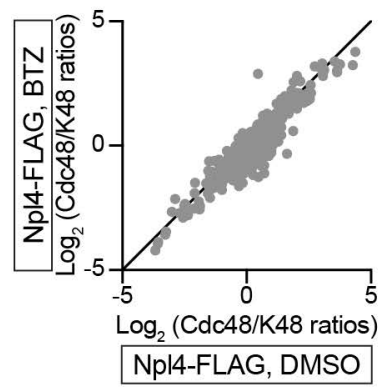

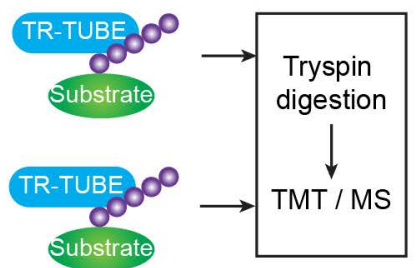

E

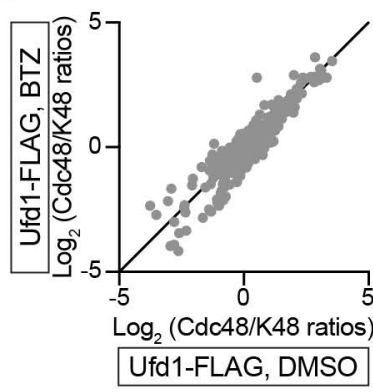

F

Distribution of the averaged ratios

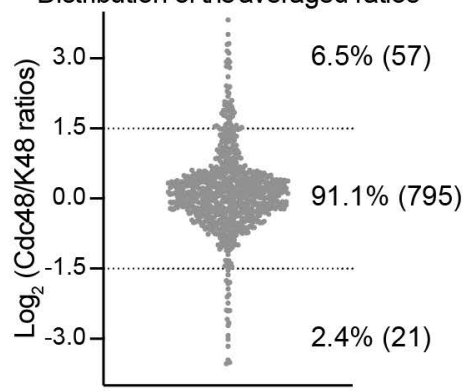


$\circ$ Streptavidin pulldown with SBP-Ufd1,

은 Npl4, Ub(n)-Dy-sfGFP, and ADP
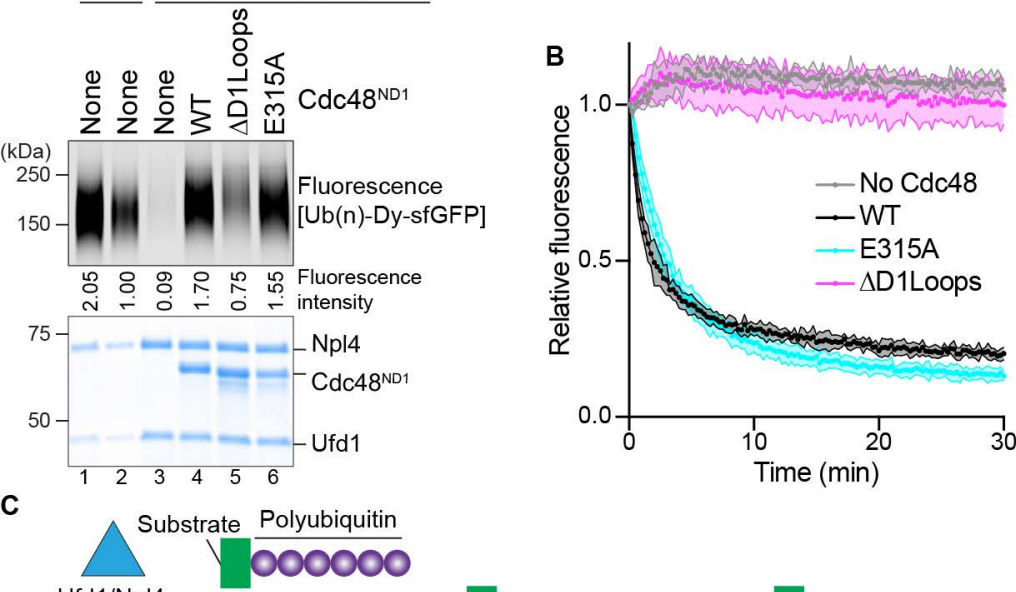

Ufd $1 / \mathrm{Npl} 4$

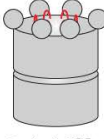

Cdc482Cys

(oxidized)
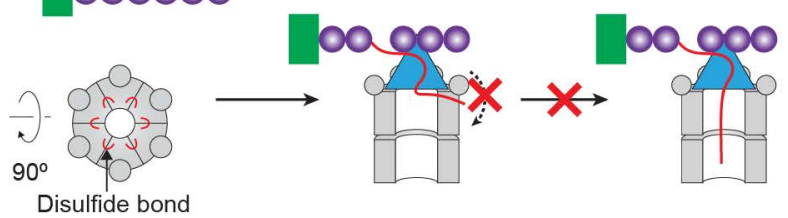

$\mathbf{F}$

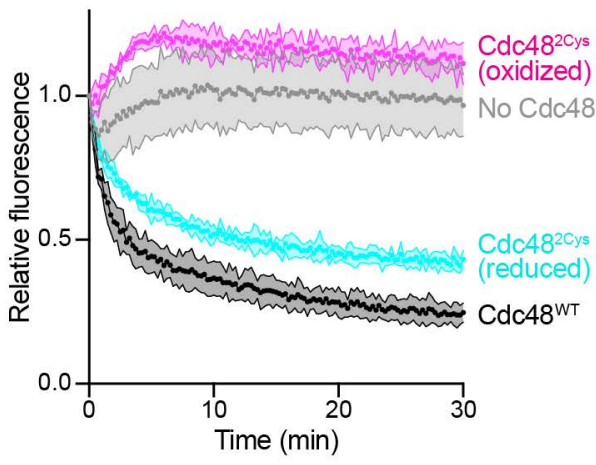

D

Anti-FLAG pulldown in ADP
$\frac{++++}{+}$ Ufd 1

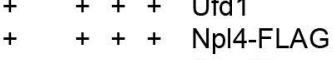

$++\quad \mathrm{Cdc} 48$

$+\quad$ Cdc48 2 Cys (oxidized)
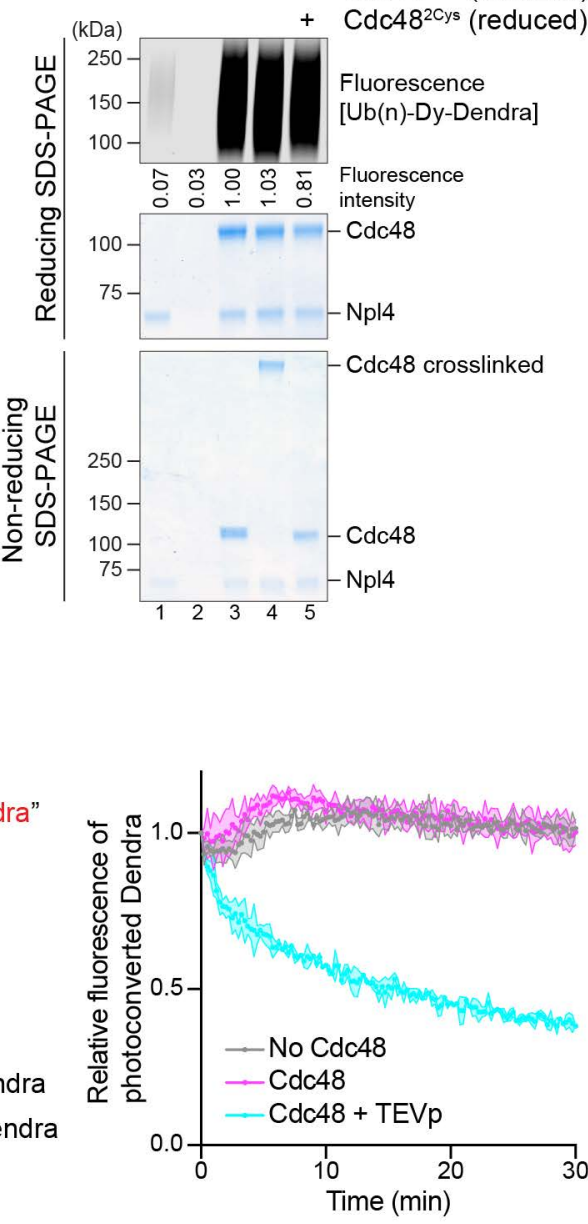

\section{Figure 3}




$$
\text { A Ub2 }
$$

Ub1 ᄂ

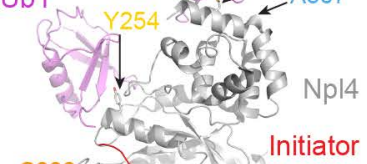
S338 4 Ub

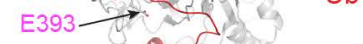

$\mathrm{K} 345 \rightarrow \delta$

Cdc48

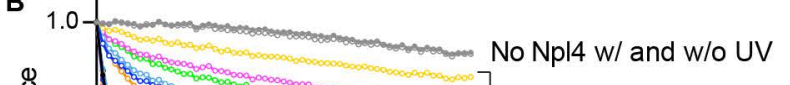

告 $\mathrm{Np} / 4^{\mathrm{Bpa}} \mathrm{W} / \mathrm{UV}$

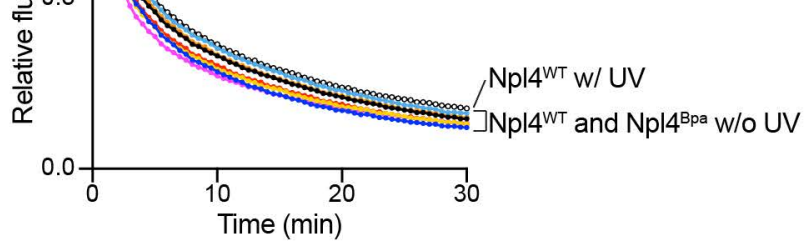

Percentage of Percentage of crosslinking unfolding inhibition

$$
\begin{aligned}
& \text { M574Bpa } \\
& \text { A567Bpa } \\
& \text { Y254Bpa } \\
& \text { S338Bpa } \\
& \text { E393Bpa } \\
& \text { K345Bpa }
\end{aligned}
$$

59.3

56.3

64.4

50.3

69.0

79.2

61.3

68.2

91.5

58.3

81.0

78.8

Figure 4 


\section{Polyubiquitin \\ Substrate}
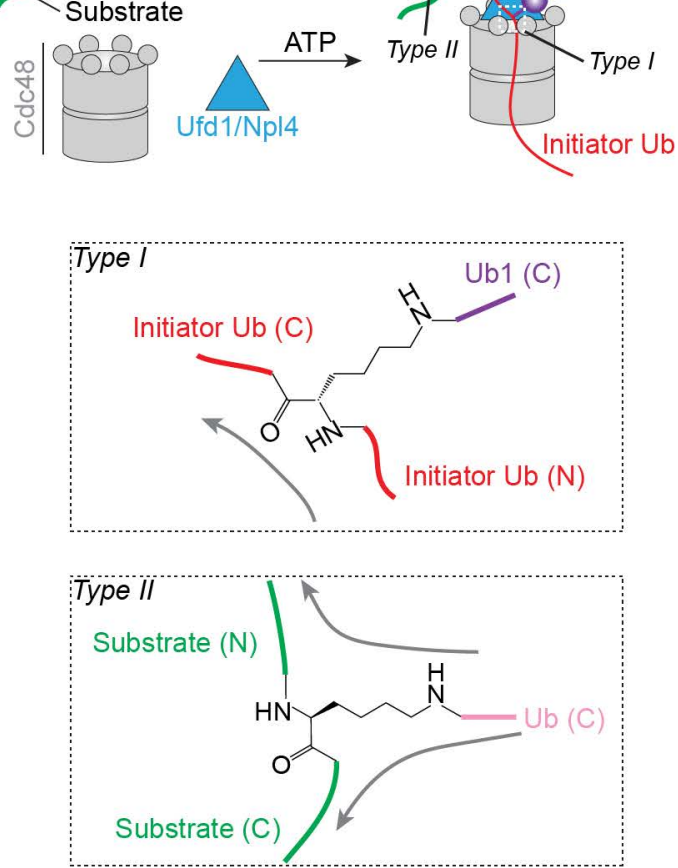

D

$$
\text { "Dendra-Ub(n)-sfGFP" }
$$
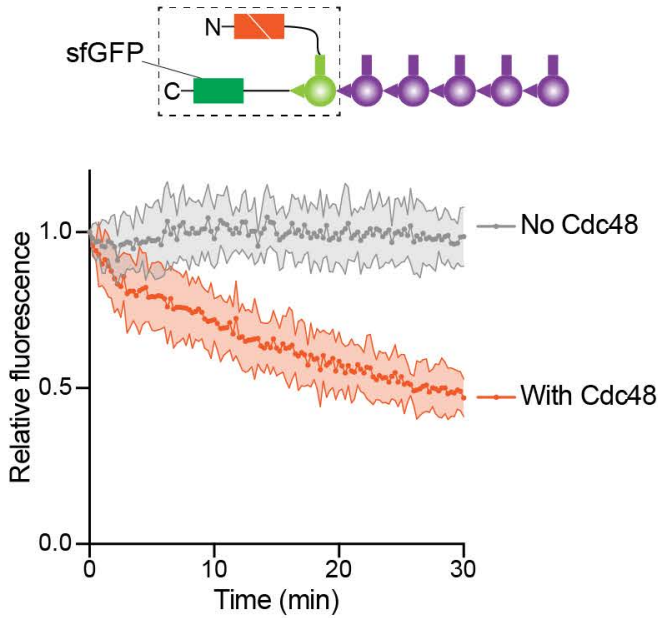

B

$\mathrm{N}$-terminus

$\mathrm{Ub}^{\mathrm{WT}}$ $U b^{K 48 R}$ C-terminus $\rightarrow-\mathrm{K}^{\mathrm{K} 48} \quad \mathrm{Ub}^{\mathrm{G} 76 \mathrm{~V}} \quad$ Dendra Dendra-Dist N 606-606.

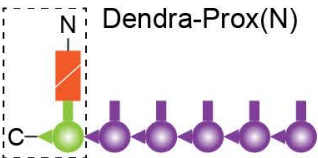

Dendra-Ub ${ }^{\mathrm{K} 48 R}$-Prox(N)

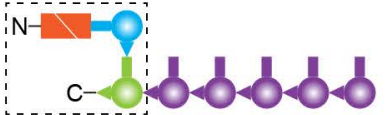

C

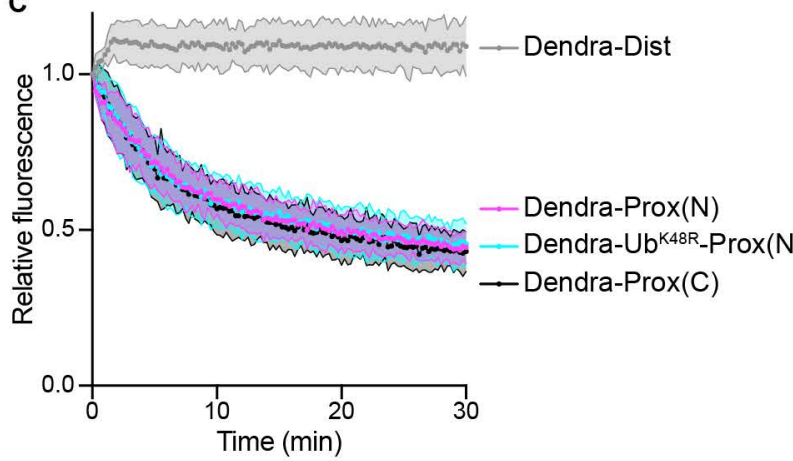

E "sfGFP-Ub(n)-Dendra"
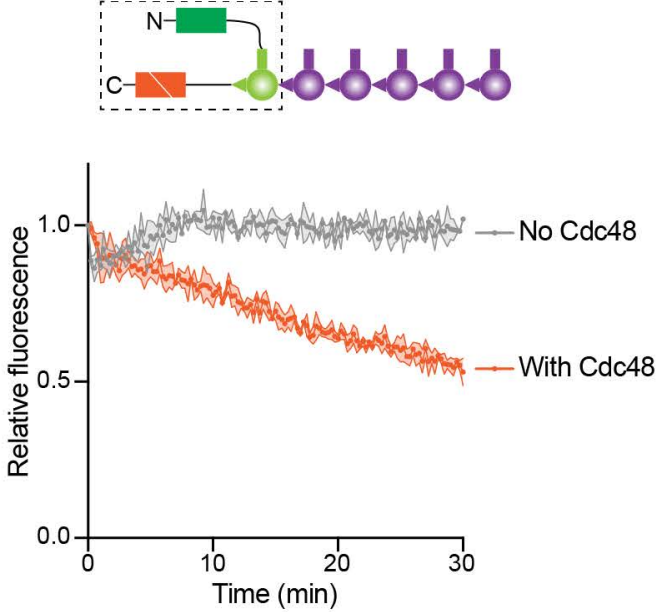
$-++\frac{1+}{-+}$ Npl4-FLAG

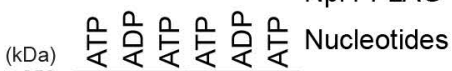
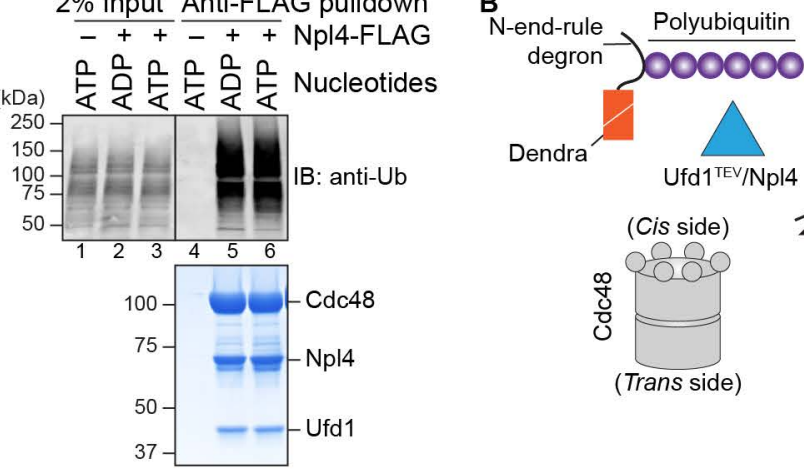

(Cis side)

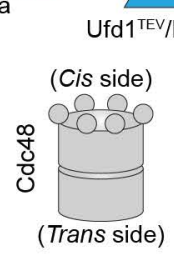

(Trans side)

FLAG pulldown with Cdc48-FLAG, $\mathrm{Npl} 4$, and Ub(n)-Dy-Dendra

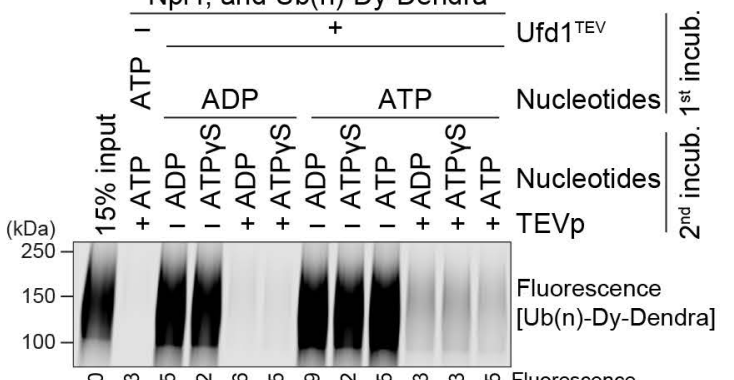

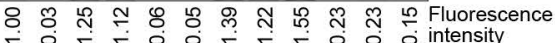

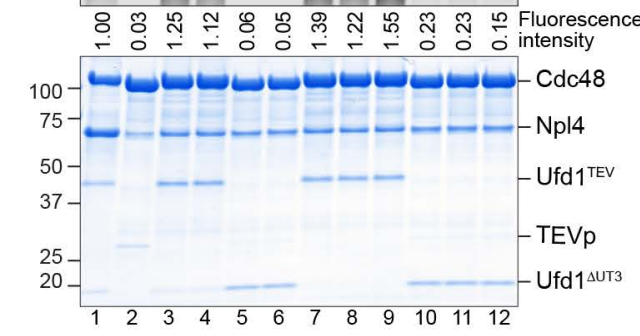

E $\mathrm{kDa}$
250 1

$12.8 \% \quad 2^{\text {nd }}$ incub. $\rightarrow$ anti-FLAG pulldown

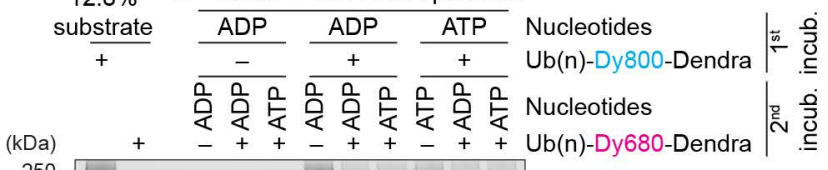

250
150
100
250
150
100

250
150
100
250
1
100
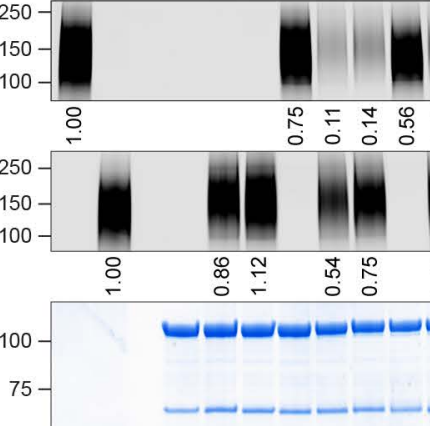

$\stackrel{5}{5} \forall \varphi$ L $\square$ Fluorescence

[Ub(n)-Dy800-Dendra]

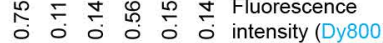

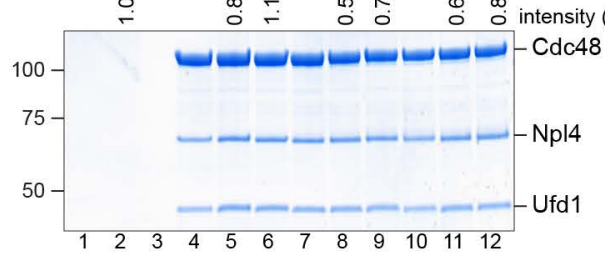

Fluorescence

[Ub(n)-Dy680-Dendra]
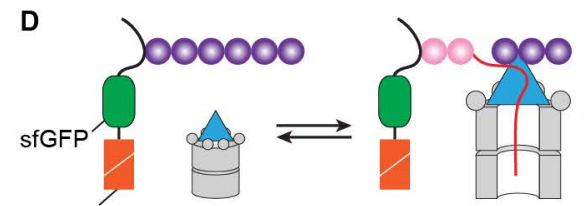

$\underset{\text { ATP }}{\stackrel{(a)}{\longrightarrow}}$

Dendra
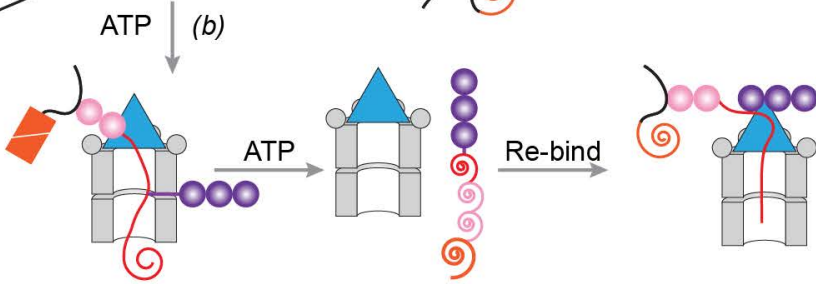

FLAG pulldown with $\mathrm{Cdc} 48$ and $15 \%$ input Ub(n)-Dy-sfGFP-Dendra

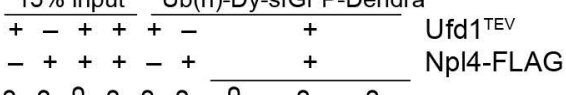

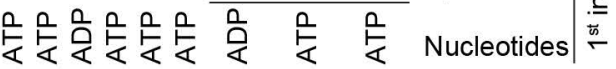
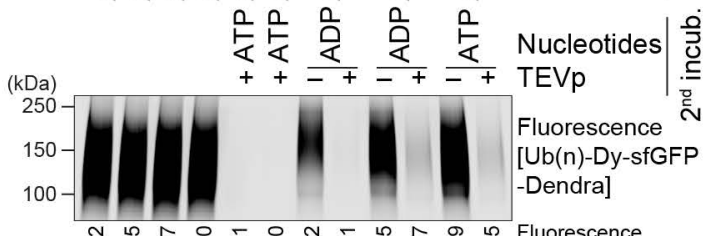

สิ

$00-10000000000$ intensity

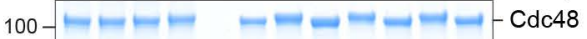
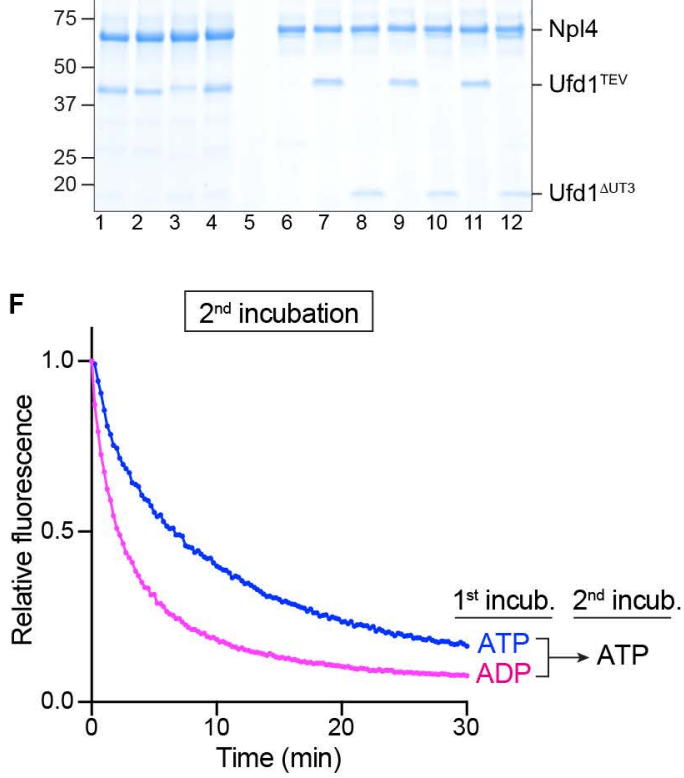
